\title{
Coupled Modified KP Hierarchy and Its Dispersionless Limit
}

\author{
Takashi TAKEBE ${ }^{\dagger}$ and Lee-Peng TEO \\ $\dagger$ Department of Mathematics, Ochanomizu University, \\ Otsuka 2-1-1, Bunkyo-ku, Tokyo, 112-8610, Japan \\ E-mail: takebe@math.ocha.ac.jp \\ $¥$ Faculty of Information Technology, Multimedia University, \\ Jalan Multimedia, Cyberjaya, 63100, Selangor Darul Ehsan, Malaysia \\ E-mail:lpteo@mmu.edu.my
}

Received August 18, 2006, in final form October 03, 2006; Published online October 31, 2006

Original article is available at http://www.emis.de/journals/SIGMA/2006/Paper072/

\begin{abstract}
We define the coupled modified KP hierarchy and its dispersionless limit. This integrable hierarchy is a generalization of the "half" of the Toda lattice hierarchy as well as an extension of the mKP hierarchy. The solutions are parametrized by a fibered flag manifold. The dispersionless counterpart interpolates several versions of dispersionless mKP hierarchy.
\end{abstract}

Key words: cmKP hierarchy; fibered flag manifold; dcmKP hierarchy

2000 Mathematics Subject Classification: 37K10

Dedicated to the memory of Vadim Kuznetsov.

\section{Introduction}

Since 1980's many integrable systems with infinitely many degrees of freedom have been studied by means of infinite dimensional homogeneous spaces. Well-known examples are: the KP hierarchy and the Sato-Grassmann manifold ([10, 12, 11, 3] etc.), the Toda lattice hierarchy and " $G L(\infty) "([23,13,15,16]$ etc.), the modified KP (mKP) hierarchy and the flag manifold ([5, 6, $2,7,17]$ etc.). In this paper we add one more example to this series: the coupled modified KP (cmKP) hierarchy and the fibered flag manifold.

The modified KP hierarchy is defined in [2] and [17] as a system consisting of two sets of equations: the Lax equations for continuous variables $t=\left(t_{1}, t_{2}, \ldots\right)$ and a set of difference equations for the discrete variable $s$. The cmKP hierarchy has the same description but the normalization of the operators is different. By this difference the moduli space of solutions of the mKP hierarchy (= the flag manifold) is enlarged.

Actually a special case of the cmKP hierarchy has been known since [23], in which the Toda lattice hierarchy was introduced. A half of the Toda lattice hierarchy without dependence on half of time variables is a cmKP hierarchy (See Appendix B). Therefore the cmKP hierarchy can be considered as the mKP hierarchy coupled to the Toda field.

In this special case the solution space is parametrized by the basic affine space $G L(\infty) / N$ where $N$ is the subgroup of infinite upper triangular matrices with unity on the diagonal. In other words it is a product of the full flag manifold and $\left(\mathbb{C}^{\times}\right)^{\mathbb{Z}}$. The solution space of our cmKP hierarchy is (partial flag manifold) $\times \prod_{s \in S^{\prime}}\left(\mathbb{C}^{m_{s}} \backslash\{0\}\right)$ in general. (See Corollary 1.)

${ }^{\star}$ This paper is a contribution to the Vadim Kuznetsov Memorial Issue "Integrable Systems and Related Topics". The full collection is available at http://www.emis.de/journals/SIGMA/kuznetsov.html 
The dispersionless (quasi-classical) limit of the cmKP hierarchy is taken in the same way as the dispersionless KP and Toda hierarchies. (See [21] and references therein.) We call the resulting system the dispersionless cmKP hierarchy (the $d c m K P$ hierarchy in short). In fact the dcmKP hierarchy was first introduced by one of the authors [18] (in a slightly different form) as a system which interpolates two versions of the dispersionless mKP hierarchies, one by [8] and [1] and the other by [17]. Hence the name "dispersionless coupled mKP hierarchy" has another interpretation: It connects variants of the dispersionless mKP hierarchy.

This paper is organized as follows: The part on the cmKP hierarchy (Section 2) follows standard recipe. We start from the Lax representation similar to that of the mKP hierarchy [17] and introduce the dressing operator and the wave function as solutions of linear problems. We show existence of the $\tau$ functions and construct them explicitly, using the DJKM free fermions.

The dispersionless counterpart is discussed in Section 3, following the strategy of [21]. The basic objects are a formal power series $\mathcal{L}$ and a polynomial $\mathcal{P}$. The hierarchy is defined by the Lax equations. Then we introduce the dressing function, the Orlov-Schulman function, the $S$ function and the $\tau$ function. We also discuss the relation with the dispersionless mKP hierarchy and the characterization of the $\tau$ function.

In Section 3.8 the cmKP hierarchy and the dcmKP hierarchy, so far discussed independently in principle, are related via the WKB analysis.

Equivalent formulations of the cmKP hierarchy are discussed in the appendices.

\section{Coupled modified KP hierarchy}

\subsection{Definition of the cmKP hierarchy}

In this section we define the cmKP hierarchy with discrete parameters $\left\{n_{s}\right\}_{s \in S} \subset \mathbb{Z}$, where $S$ is a set of consecutive integers (e.g., $S=\mathbb{Z}, S=\{0,1, \ldots, n\}$ etc.) as in [17]. The dispersionless limit can be taken only when $S=\mathbb{Z}, n_{s}=N s$. Set $S^{\prime}=S \backslash\{$ maximum element of $S\}$ if there exists a maximum element of $S$ and $S^{\prime}=S$ otherwise.

The independent variables of the cmKP hierarchy are the discrete variable $s \in S$ and the set of continuous variables $t=\left(t_{1}, t_{2}, \ldots\right)$. The dependent variables are encapsulated in the following operators with respect to $x$ :

$$
\begin{aligned}
L(s ; x, t) & =\partial+u_{1}(s, x, t)+u_{2}(s, x, t) \partial^{-1}+\cdots \\
& =\sum_{n=0}^{\infty} u_{n}(s, x, t) \partial^{1-n}, \\
P(s ; x, t) & =p_{0}(s, x, t) \partial^{m_{s}}+\cdots+p_{m_{s}-1}(s, x, t) \partial \\
& =\sum_{n=0}^{m_{s}-1} p_{n}(s, x, t) \partial^{m_{s}-n} .
\end{aligned}
$$

where $\partial=\partial_{x}, u_{0}=1, p_{0} \neq 0, m_{s}:=n_{s+1}-n_{s} . P(s ; x, t)$ is defined only for $s \in S^{\prime}$. We often write $L(s), P(s)$ instead of $L(s ; x, t), P(s ; x, t)$. The notation $(L(s), P(s))_{s \in S}$ stands for a pair of sequences $\left((L(s))_{s \in S},(P(s))_{s \in S^{\prime}}\right)$.

The cmKP hierarchy is the following system of differential and difference equations:

$$
\begin{aligned}
& \frac{\partial L(s)}{\partial t_{n}}=\left[B_{n}(s), L(s)\right], \\
& L(s+1) P(s)=P(s) L(s), \\
& \left(\frac{\partial}{\partial t_{n}}-B_{n}(s+1)\right) P(s)=P(s)\left(\frac{\partial}{\partial t_{n}}-B_{n}(s)\right),
\end{aligned}
$$


where $B_{n}(s)=B_{n}(s ; x, t)=\left(L(s ; x, t)^{n}\right)_{>0}$. The projections like $(\cdot)_{>0}$ are defined as follows: for $A(x, \partial)=\sum_{n \in \mathbb{Z}} a_{n}(x) \partial^{n}$,

$$
\begin{array}{ll}
A_{>0}:=\sum_{n>0} a_{n}(x) \partial^{n}, & A_{\geq 0}:=\sum_{n \geq 0} a_{n}(x) \partial^{n}, \\
A_{<0}:=\sum_{n<0} a_{n}(x) \partial^{n}, & A_{\leq 0}:=\sum_{n \leq 0} a_{n}(x) \partial^{n} .
\end{array}
$$

The last equation (2.5) can be written in the form

$$
\frac{\partial P(s)}{\partial t_{n}}=B_{n}(s+1) P(s)-P(s) B_{n}(s),
$$

as well. Since $B_{1}(s)=\partial$, equations (2.3) and (2.6) for $n=1$ imply that $x$ and $t_{1}$ always appear in the combination $x+t_{1}$.

Note that the cmKP hierarchy is almost the same as the mKP hierarchy in [2] or [17] but the forms of $L(s), P(s)$ and $B_{n}(s)$ are different.

Remark 1. We can start from $P(s)$ with the 0 -th order terms, but if $(L(s), P(s))_{s \in \mathbb{Z}}$ satisfies the cmKP hierarchy, we can gauge away such terms. See Appendix A for details.

By the well-known argument (cf. [3, $\S 1]$, [23, Theorem 1.1]) we can prove that the Lax equations (2.3) is equivalent to the Zakharov-Shabat (or zero-curvature) equations,

$$
\left[\frac{\partial}{\partial t_{m}}-B_{m}(s), \frac{\partial}{\partial t_{n}}-B_{n}(s)\right]=0
$$

or

$$
\left[\frac{\partial}{\partial t_{m}}-B_{m}^{c}(s), \frac{\partial}{\partial t_{n}}-B_{n}^{c}(s)\right]=0
$$

where $B_{n}^{c}(s):=-\left(L(s)^{n}\right)_{\leq 0}=B_{n}(s)-L(s)^{n}$.

\subsection{Dressing operator, wave function}

Similarly to the mKP hierarchy, we can show the existence of the dressing operator.

Proposition 1. For any solution $(L(s), P(s))_{s \in S}$ there exists an operator $W(s)=W(s ; x, t ; \partial)$ of the form

$$
W(s ; x, t ; \partial)=\left(w_{0}(s ; x, t)+w_{1}(s ; x, t) \partial^{-1}+\cdots\right) \partial^{n_{s}}, \quad w_{0}(s ; x, t) \neq 0,
$$

satisfying equations

$$
L(s) W(s)=W(s) \partial, \quad P(s) W(s)=W(s+1), \quad \frac{\partial W(s)}{\partial t_{n}}=B_{n}^{c}(s) W(s) .
$$

In fact, equations (2.3), (2.8), (2.4) and (2.5) are compatibility conditions for the linear system (2.10).

We call $W(s ; x, t ; \partial)$ the dressing operator.

The wave function $w(s ; \lambda)=w(s ; x, t ; \lambda)$ is defined by

$$
\begin{aligned}
w(s ; x, t ; \lambda) & :=W(s ; x, t ; \partial) e^{\xi(x+t, \lambda)} \\
& =\left(\sum_{j=0}^{\infty} w_{j}(s ; x, t) \lambda^{-j}\right) \lambda^{n_{s}} e^{\xi(x+t, \lambda)},
\end{aligned}
$$


where $x+t:=\left(x+t_{1}, t_{2}, t_{3}, \ldots\right), \xi(x+t, \lambda)=x \lambda+\sum_{n=1}^{\infty} t_{n} \lambda^{n}$. It is subject to the equations:

$$
\begin{aligned}
& L(s) w(s ; \lambda)=\lambda w(s ; \lambda), \\
& \frac{\partial}{\partial t_{n}} w(s ; \lambda)=B_{n}(s) w(s ; \lambda), \\
& P(s) w(s ; \lambda)=w(s+1 ; \lambda) .
\end{aligned}
$$

Recall that the mKP hierarchy in [17] is defined by the equations (2.3)-(2.5) for operators $L(s)=L^{\mathrm{mKP}}(s), B_{n}(s)=B_{n}^{\mathrm{mKP}}(s), P(s)=P^{\mathrm{mKP}}(s)$ normalized as

$$
\begin{aligned}
& L^{\mathrm{mKP}}(s ; x, t)=\partial+u_{2}^{\mathrm{mKP}}(s, x, t) \partial^{-1}+u_{3}^{\mathrm{mKP}}(s, x, t) \partial^{-2}+\cdots, \\
& B_{n}^{\mathrm{mKP}}(s ; x, t):=\left(L^{\mathrm{mKP}}(s ; x, t)^{n}\right)_{\geq 0}, \\
& P^{\mathrm{mKP}}(s ; x, t)=\partial^{m_{s}}+q_{1}(s, x, t) \partial^{m_{s}-1}+\cdots+q_{m_{s}}(s, x, t) .
\end{aligned}
$$

Its dressing operator $W(s)=W^{\mathrm{mKP}}(s)$ is normalized as

$$
W^{\mathrm{mKP}}(s)=\left(1+w_{1}^{\mathrm{mKP}}(s) \partial^{-1}+\cdots\right) \partial^{n_{s}},
$$

and satisfies the same linear equations (2.10) as the cmKP hierarchy, where $B_{n}^{c}(s)=B_{n}^{\mathrm{mKP}, c}(s)=$ $-\left(L^{\mathrm{mKP}}(s)^{n}\right)_{<0}=B_{n}^{\mathrm{mKP}}(s)-L^{\mathrm{mKP}}(s)^{n}$.

Proposition 2. (i) Let $(L(s), P(s))_{s \in S}$ be a solution of the cmKP hierarchy and $W(s)$ be the corresponding dressing operator of the form (2.9). Then $\left(L^{\mathrm{mKP}}(s), P^{\mathrm{mKP}}(s)\right)_{s \in S}$ defined by

$$
L^{\mathrm{mKP}}(s):=w_{0}(s)^{-1} L(s) w_{0}(s), \quad P^{\mathrm{mKP}}(s):=w_{0}(s+1)^{-1} P(s) w_{0}(s)
$$

is a solution of the $m K P$ hierarchy and $W^{\mathrm{mKP}}(s):=w_{0}(s)^{-1} W(s)$ is the corresponding dressing operator.

(ii) Conversely, if a sequence $\left\{\left(f^{(0)}(s), \ldots, f^{\left(m_{s}-1\right)}(s)\right)\right\}_{s \in S}$ of non-zero constant vectors and a solution of the $m K P$ hierarchy $\left(L^{\mathrm{mKP}}(s), P^{\mathrm{mKP}}(s)\right)_{s \in S}$ are given, there exists a unique function $f(s)=f(s ; x, t)$ such that

$$
\partial^{k} f(s ; 0,0)=f^{(k)}(s) \quad \text { for all } \quad s \in S, 0 \leqq k<m_{s},
$$

and $\left(L(s):=f(s)^{-1} L^{\mathrm{mKP}}(s) f(s), P(s):=f(s+1)^{-1} P^{\mathrm{mKP}}(s) f(s)\right)_{s \in S}$ is a solution of the cmKP hierarchy. $\left(m_{\max (S)}=\infty\right.$.)

Proof. (i) Note that the linear equations (2.10) imply that

$$
\begin{aligned}
& \frac{\partial}{\partial x} w_{0}(s ; x, t)=-u_{1}(s ; x, t) w_{0}(s ; x, t), \\
& w_{0}(s ; x, t) p_{0}(s ; x, t)=w_{0}(s+1 ; x, t), \\
& \frac{\partial}{\partial t_{n}} w_{0}(s ; x, t)=-\left(L(s)^{n}\right)_{0} w_{0}(s ; x, t) .
\end{aligned}
$$

Equation (2.18) and (2.19) mean that $L^{\mathrm{mKP}}(s)=w_{0}(s)^{-1} L(s) w_{0}(s)$ and $P^{\mathrm{mKP}}(s)=w_{0}(s+$ $1)^{-1} P(s) w_{0}(s)$ have the required form (2.13) and (2.15). It follows from equation (2.20) that

$$
w_{0}(s)^{-1}\left(\frac{\partial}{\partial t_{n}}-B_{n}(s)\right) w_{0}(s)=\frac{\partial}{\partial t_{n}}-B_{n}^{\mathrm{mKP}}(s),
$$

where $B_{n}^{\mathrm{mKP}}(s)$ is defined by $(2.14)$ from $L^{\mathrm{mKP}}(s)$. It is easy to see that $\left(L^{\mathrm{mKP}}(s), P^{\mathrm{mKP}}(s)\right)_{s \in S}$ satisfies the system $(2.3)-(2.5)$.

(ii) is proved in almost the same way as the fact mentioned in Remark 1, so we prove it in Appendix A. 
It was shown in [17] that dressing operators of the mKP hierarchy are parametrized by the flag manifold: Let $\mathcal{V}$ be an infinite dimensional linear space $\bigoplus_{\nu \in \mathbb{Z}} \mathbb{C} e_{\nu}$ with basis $\left\{e_{\nu}\right\}_{\nu \in \mathbb{Z}}$ and $V^{\varnothing}$ be its subspace defined by $V^{\varnothing}=\bigoplus_{\nu \geq 0} \mathbb{C} e_{\nu}$. (Actually we have to take completion of $\mathcal{V}$, but details are omitted.) The Sato-Grassmann manifold of charge $n, S G M^{(n)}$, is defined by

$$
S G M^{(n)}=\left\{U \subset \mathcal{V} \mid \text { index of } U \rightarrow \mathcal{V} / V^{\varnothing} \text { is } n\right\} .
$$

The set of dressing operators of the KP hierarchy is $S G M^{(0)}$ as is shown in $[10,12]$ or [11] and the set of dressing operators of the mKP hierarchy is the flag manifold

$$
\text { Flag }:=\left\{\left(U_{s}\right)_{s \in S} \mid U_{s} \in S G M^{\left(n_{s}\right)}, U_{s} \subset U_{s+1}\right\} .
$$

See Proposition 1.3 of [17]. Hence the set of the dressing operators of the cmKP hierarchy is described as follows.

Corollary 1. The dressing operator of the cmKP hierarchy $(W(s))_{s \in S}$ is parametrized by Flag $\times$ $\prod_{s \in S^{\prime}}\left(\mathbb{C}^{m_{s}} \backslash\{0\}\right)$.

Schematically, this space is an infinite dimensional homogeneous space $G L(\infty) / Q$, where $Q$ is a subgroup of the group $G L(\infty)$ of invertible $\mathbb{Z} \times \mathbb{Z}$ matrices defined as follows: $g=\left(g_{i j}\right)_{i, j \in \mathbb{Z}} \in Q$ if and only if

$$
g_{i j}= \begin{cases}0, & i>n_{s}, j \leqq n_{s} \text { or } i>n_{s}+1, j \leqq n_{s}+1 \text { for some } s \in S, \\ 1, & i=j=n_{s}+1 \text { for some } s \in S\end{cases}
$$

In fact, if we consider an intermediate parabolic subgroup $P$ defined by

$$
g=\left(g_{i j}\right)_{i, j \in \mathbb{Z}} \in P \Longleftrightarrow g_{i j}=0 \quad\left(i>n_{s}, j \leqq n_{s} \text { for some } s \in S\right),
$$

$G L(\infty) / Q$ is considered as the fiber bundle

$$
G L(\infty) / Q \rightarrow G L(\infty) / P
$$

over $G L(\infty) / P=$ Flag. A point on a fiber $\left(U_{s}\right)_{s \in S} \in$ Flag specifies a series of non-zero vectors in $U_{s+1} / U_{s}(s \in S) .\left(U_{\max (S)+1}=\mathcal{V}\right.$. $)$

We do not go into details of infinite dimensional homogeneous spaces. In this picture it is clear that the group $G L(\infty)$ acts on the space of solutions transitively. The action is explicitly described in terms of the fermionic description of the $\tau$ functions. See the end of Section 2.5.

\subsection{Bilinear identity}

Recall that the wave function and the adjoint wave function of the mKP hierarchy have the form

$$
\begin{aligned}
& w^{\mathrm{mKP}}(s ; x, t ; \lambda):=W^{\mathrm{mKP}}(s) e^{\xi(x+t ; \lambda)}=\hat{w}^{\mathrm{mKP}}(s ; x, t ; \lambda) \lambda^{n_{s}} e^{\xi(x+t ; \lambda)}, \\
& \hat{w}^{\mathrm{mKP}}(s ; x, t ; \lambda):=1+w_{1}^{\mathrm{mKP}}(s ; x, t) \lambda^{-1}+\cdots,
\end{aligned}
$$

and

$$
\begin{aligned}
& w^{\mathrm{mKP}, *}(s ; x, t ; \lambda):=\left(\left(W(s)^{\mathrm{mKP}}\right)^{*}\right)^{-1} e^{-\xi(x+t ; \lambda)}=\hat{w}^{\mathrm{mKP}, *}(s ; x, t ; \lambda) \lambda^{-n_{s}} e^{-\xi(x+t ; \lambda)}, \\
& \hat{w}^{\mathrm{mKP}, *}(s ; x, t ; \lambda):=1+w_{1}^{\mathrm{mKP}, *}(s ; x, t) \lambda^{-1}+\cdots,
\end{aligned}
$$


where $A^{*}$ for an operator $A$ denotes its formal adjoint: $x^{*}=x, \partial^{*}=-\partial,(A B)^{*}=B^{*} A^{*}$. These functions are characterized by the bilinear residue identity:

$$
\operatorname{Res}_{\lambda=\infty} w^{\mathrm{mKP}}(s ; x, t ; \lambda) w^{\mathrm{mKP}, *}\left(s^{\prime} ; x^{\prime}, t^{\prime} ; \lambda\right) d \lambda=0,
$$

for any $x, t, x^{\prime}, t^{\prime}$ and $s^{\prime} \leqq s$. See $\S 1$ of [17] for details.

The wave function of the cmKP hierarchy is also characterized by a bilinear identity as follows:

Proposition 3. (i) The wave function of the cmKP hierarchy satisfies the following identity:

$$
\operatorname{Res}_{\lambda=\infty} w(s ; x, t ; \lambda) \tilde{w}\left(s^{\prime} ; x^{\prime}, t^{\prime} ; \lambda\right) d \lambda= \begin{cases}0, & s^{\prime}<s \\ 1, & s^{\prime}=s .\end{cases}
$$

Here $\tilde{w}(s, t ; \lambda)$ is the adjoint wave function defined by

$$
\begin{aligned}
& \tilde{w}(s ; x, t ; \lambda):=\tilde{W}(s ; x, t ; \partial) e^{-\xi(x+t, \lambda)}, \\
& \tilde{W}(s, t ; \partial)=-\partial^{-1}\left(W(s, t ; \partial)^{-1}\right)^{*} .
\end{aligned}
$$

(ii) Conversely, let $w(s ; x, t ; \lambda)$ be a function of the form $(2.11)$ and $\tilde{w}(s ; x, t ; \lambda)$ be a function of the form (2.29), where the operator $\tilde{W}(s)$ has the form

$$
\tilde{W}(s ; x, t ; \partial)=\left(\tilde{w}_{0}(s ; x, t)+\tilde{w}_{1}(s ; x, t)(-\partial)^{-1}+\cdots\right)(-\partial)^{-n_{s}-1} .
$$

If the pair $(w(s ; x, t ; \lambda), \tilde{w}(s ; x, t ; \lambda))$ satisfies the equation $(2.28)$, then $w(s ; x, t ; \lambda)$ is a wave function of the cmKP hierarchy and $\tilde{w}(s ; x, t ; \lambda)$ is its adjoint.

Proof. (i) When $s^{\prime}=s$, we have only to show

$$
\operatorname{Res}_{\lambda=\infty} \frac{\partial^{\alpha} w}{\partial t^{\alpha}}(s ; x, t ; \lambda) \tilde{w}\left(s ; x^{\prime}, t ; \lambda\right) d \lambda= \begin{cases}1, & \alpha=(0,0, \ldots), \\ 0, & \text { otherwise }\end{cases}
$$

for each multi-index $\alpha=\left(\alpha_{1}, \alpha_{2}, \ldots\right)$.

Let us recall DJKM's lemma (Lemma 1.1 of [3]): For any operators $A\left(x, \partial_{x}\right)$ and $B\left(x, \partial_{x}\right)$, we have

$$
\operatorname{Res}_{\lambda=\infty} A\left(x, \partial_{x}\right) e^{x \lambda} B\left(x^{\prime}, \partial_{x^{\prime}}\right) e^{-x^{\prime} \lambda} d \lambda=f\left(x, x^{\prime}\right),
$$

where $f\left(x, x^{\prime}\right)$ is determined by

$$
f\left(x, x^{\prime}\right) \partial^{-1} \delta\left(x-x^{\prime}\right)=\left(A\left(x, \partial_{x}\right) B^{*}\left(x, \partial_{x}\right)\right)_{<0} \delta\left(x-x^{\prime}\right) .
$$

Since $W(s ; \partial) \tilde{W}(s ; \partial)^{*}=\partial^{-1}$ by the definition $(2.30)$ of $\tilde{W}(s ; \partial)$, we have $(2.32)$ for $\alpha=$ $(0,0, \ldots)$ thanks to $(2.33)$.

When $\alpha \neq(0,0, \ldots)$, we can prove by induction that

$$
\frac{\partial^{\alpha}}{\partial t^{\alpha}} w(s ; x, t ; \lambda)=\sum_{i \geq 1} c_{i}^{(\alpha)}(s ; x, t) \partial^{i} w(s ; x, t ; \lambda),
$$

where $c_{i}^{(\alpha)}$ 's are differential polynomials of coefficients of $B_{n}(s)$ 's. Hence the left hand side of (2.32) vanishes due to (2.33) because

$$
\left(\sum_{i \geq 1} c_{i}^{(\alpha)}(s ; x, t) \partial^{i}\right) W(s ; x, t ; \partial) \tilde{W}(s ; x, t ; \partial)^{*}=\sum_{i \geq 1} c_{i}^{(\alpha)}(s ; x, t) \partial^{i-1}
$$

is a differential operator. 
When $s^{\prime}<s$, the bilinear residue identity (2.28) is equivalent to the vanishing of its Taylor coefficients:

$$
\operatorname{Res}_{\lambda=\infty} \frac{\partial^{\alpha} w}{\partial t^{\alpha}}(s ; x, t ; \lambda) \tilde{w}\left(s^{\prime} ; x^{\prime}, t ; \lambda\right) d \lambda=0,
$$

for each multi-index $\alpha$. When $\alpha=(0,0, \ldots)$, this follows directly from (2.33) since

$$
\begin{aligned}
W(s ; \partial) \tilde{W}\left(s^{\prime} ; \partial\right)^{*} & =W(s ; \partial) W\left(s^{\prime} ; \partial\right)^{-1} \partial^{-1} \\
& =P(s-1) P(s-2) \cdots P\left(s^{\prime}+1\right) P\left(s^{\prime}\right) \partial^{-1},
\end{aligned}
$$

which is a differential operator due to $(2.2)$. For $\alpha \neq(0,0, \ldots)$, the proof is similar to the case $s^{\prime}=s$.

(ii) When $s^{\prime}=s$ and $t=t^{\prime}$, the bilinear residue identity (2.28) is equivalent to

$$
\left(W(s ; x, t ; \partial) \tilde{W}(s ; x, t ; \partial)^{*}\right)_{<0}=\partial^{-1},
$$

which means

$$
W(s ; x, t ; \partial) \tilde{W}(s ; x, t ; \partial)^{*}=\partial^{-1},
$$

because $W(s ; x, t ; \partial) \tilde{W}(s ; x, t ; \partial)^{*}$ is of order -1 . Hence

$$
\tilde{W}(s ; x, t ; \partial)=-\partial^{-1}\left(W(s ; x, t ; \partial)^{-1}\right)^{*} .
$$

Putting $s^{\prime}=s-1$ and $t=t^{\prime}$, we have

$$
\left(W(s ; x, t ; \partial) \tilde{W}(s-1 ; x, t ; \partial)^{*}\right)_{<0}=0 .
$$

from (2.28). This means

$$
W(s ; x, t ; \partial) W(s-1 ; x, t ; \partial)^{-1} \partial^{-1}=(\text { differential operator }),
$$

by (2.37). Hence we obtain

$$
\begin{aligned}
P(s-1 ; \partial) & :=W(s, t ; \partial) W(s-1, t ; \partial)^{-1} \\
& =\left(\text { differential operator of order } m_{s-1} \text { divisible by } \partial\right) .
\end{aligned}
$$

Finally, put $s^{\prime}=s$ and differentiate (2.28) with respect to $t_{n}$. Then we have

$$
\operatorname{Res}_{\lambda=\infty} \frac{\partial}{\partial t_{n}} w(s, t ; \lambda) \tilde{w}(s, t ; \lambda) d \lambda=0
$$

namely,

$$
\left(\left(\frac{\partial W(s)}{\partial t_{n}}+W(s) \partial^{n}\right) \tilde{W}(s)^{*}\right)_{<0}=0
$$

Using (2.37), we can rewrite this equation as

$$
\frac{\partial W(s)}{\partial t_{n}} W(s)^{-1}=-\left(W(s) \partial^{n} W(s)^{-1}\right)_{\leq 0} .
$$

Thus we have recovered the linear equations (2.10).

Corollary 2. The function $w^{\mathrm{mKP}}(s ; x, t ; \lambda):=w(s ; x, t ; \lambda) / w_{0}(s ; x, t)$ is a wave function of the $m K P$ hierarchy. Its adjoint wave function is

$$
w^{\mathrm{mKP}, *}(s ; x, t ; \lambda):=-w_{0}(s ; x, t) \partial(\tilde{w}(s ; x, t ; \lambda)) .
$$

Proof. This can be directly deduced from Proposition 2. Alternatively we derive it from Proposition 3 here. Functions $w(s ; x, t ; \lambda) / w_{0}(s ; x, t)$ and $-w_{0}(s ; x, t) \partial(\tilde{w}(s ; x, t ; \lambda))$ are expanded with respect to $\lambda$ as in (2.25) and in (2.26) respectively. Hence differentiating (2.28) with respect to $x^{\prime}$, we obtain the bilinear residue identity $(2.27)$ for the mKP hierarchy. 


\section{$2.4 \tau$ function}

In this subsection we prove that the wave functions of the cmKP hierarchy are ratios of the $\tau$ functions. In contrast to the (m)KP hierarchy, we need two series of $\tau$ functions to express the wave function, unless $m_{s}=1$.

Theorem 1. (i) Let $w(s ; x, t ; \lambda)$ be a wave function of the cmKP hierarchy and $\tilde{w}(s ; x, t ; \lambda)$ be its adjoint. Then there exist functions $\tau_{0}(s ; t)$ and $\tau_{1}(s ; t)$ such that

$$
\begin{aligned}
w(s ; x, t ; \lambda) & :=\frac{\tau_{0}\left(s ; x+t-\left[\lambda^{-1}\right]\right)}{\tau_{1}(s ; x+t)} \lambda^{n_{s}} e^{\xi(x+t ; \lambda),} \\
\tilde{w}(s ; x, t ; \lambda) & :=\frac{\tau_{1}\left(s ; x+t+\left[\lambda^{-1}\right]\right)}{\tau_{0}(s ; x+t)} \lambda^{-n_{s}-1} e^{-\xi(x+t ; \lambda)},
\end{aligned}
$$

where $\left[\lambda^{-1}\right]:=\left(\lambda^{-1}, \lambda^{-2} / 2, \lambda^{-3} / 3, \ldots\right)$. The $\tau$ functions $\tau_{0}(s ; t)$ and $\tau_{1}(s ; t)$ are determined only up to multiplication by an arbitrary function of $s$.

(ii) If $m_{s}=n_{s+1}-n_{s}=1$, we can choose $\tau$ functions so that $\tau_{1}(s ; t)=\tau_{0}(s+1 ; t)$.

(iii) The $\tau$ functions are characterized by the following bilinear residue identity:

$$
\begin{gathered}
\operatorname{Res}_{\lambda=\infty} \tau_{0}\left(s ; t-\left[\lambda^{-1}\right]\right) \tau_{1}\left(s^{\prime} ; t^{\prime}+\left[\lambda^{-1}\right]\right) e^{\xi(t, \lambda)-\xi\left(t^{\prime}, \lambda\right)} \lambda^{n_{s}-n_{s^{\prime}}-1} d \lambda \\
= \begin{cases}0, & s^{\prime}<s, \\
\tau_{1}(s ; t) \tau_{0}\left(s ; t^{\prime}\right), & s^{\prime}=s .\end{cases}
\end{gathered}
$$

Proof. (i) Let us denote the non-trivial parts of the wave function (2.11) and the adjoint wave function (2.29) as follows:

$$
\begin{aligned}
& \hat{w}(s ; x, t ; \lambda):=w_{0}(s ; x, t)+w_{1}(s ; x, t) \lambda^{-1}+w_{2}(s ; x, t) \lambda^{-2}+\cdots, \\
& \hat{\tilde{w}}(s ; x, t ; \lambda):=\tilde{w}_{0}(s ; x, t)+\tilde{w}_{1}(s ; x, t) \lambda^{-1}+\tilde{w}_{2}(s ; x, t) \lambda^{-2}+\cdots .
\end{aligned}
$$

Namely,

$$
\begin{aligned}
& w(s ; x, t ; \lambda)=\hat{w}(s ; x, t ; \lambda) \lambda^{n_{s}} e^{\xi(x+t ; \lambda)}, \\
& \tilde{w}(s ; x, t ; \lambda)=\hat{\tilde{w}}(s ; x, t ; \lambda) \lambda^{-n_{s}-1} e^{-\xi(x+t ; \lambda)} .
\end{aligned}
$$

Putting $s=s^{\prime}, x=x^{\prime}=0$, replacing $t_{n}$ by $t_{n}+\zeta^{-n} / n, t_{n}^{\prime}$ by $t_{n}$ in the bilinear identity (2.28), we have

$$
\begin{aligned}
1 & =\operatorname{Res}_{\lambda=\infty}\left(\hat{w}\left(s ; t+\left[\zeta^{-1}\right] ; \lambda\right) \hat{\tilde{w}}(s ; t ; \lambda) \frac{\lambda^{-1}}{1-\lambda \zeta^{-1}}\right) d \lambda \\
& =\hat{w}\left(s ; t+\left[\zeta^{-1}\right] ; \zeta\right) \hat{\tilde{w}}(s ; t ; \zeta) .
\end{aligned}
$$

In the limit $\zeta^{-1} \rightarrow 0$ we have

$$
w_{0}(s ; t) \tilde{w}_{0}(s ; t)=1 \text {. }
$$

Since $w_{0}(s ; t)^{-1} w(s ; t ; \lambda)$ is a wave function of the mKP hierarchy (cf. Corollary 2), there exists a tau function $\tau_{0}(s ; t)$ such that

$$
\frac{\hat{w}(s ; t ; \lambda)}{w_{0}(s, t)}=\frac{\tau_{0}\left(s ; t-\left[\lambda^{-1}\right]\right)}{\tau_{0}(s ; t)} .
$$


Define the function $\tau_{1}(s ; t)$ by

$$
\tau_{1}(s ; t):=\frac{\tau_{0}(s ; t)}{w_{0}(s ; t)}
$$

Equation (2.39) follows from (2.44) and (2.45). Equation (2.40) follows from (2.42).

(ii) From (2.40) and (2.42), we can see that dependence of $\tau_{0}(s ; t)$ on $t_{n}(n \geq 1)$ is determined uniquely by the equation

$$
\frac{\partial \log \tau_{0}(s ; t)}{\partial t_{n}}=A_{n}(s ; t)
$$

where

$$
A_{n}(s ; t)=-\operatorname{Res}_{\lambda=\infty}\left(\lambda^{n}\left(\sum_{j=1}^{\infty} \lambda^{-j-1} \frac{\partial}{\partial t_{j}}+\frac{\partial}{\partial \lambda}\right) \log \hat{\tilde{w}}(s ; t ; \lambda)\right) d \lambda .
$$

See $\S 1.6$ of [3] for detailed arguments.

When $n_{s+1}=n_{s}+1$, by putting $s^{\prime}=s-1$ and replacing $t_{n}$ by $t_{n}+\zeta^{-n} / n, t_{n}^{\prime}$ by $t_{n}$ in the bilinear identity (2.28), we have

$$
\begin{aligned}
0 & =\operatorname{Res}_{\lambda=\infty}\left(\hat{w}\left(s ; t+\left[\zeta^{-1}\right] ; \lambda\right) \hat{\tilde{w}}(s-1 ; t ; \lambda) \frac{1}{1-\lambda \zeta^{-1}}\right) d \lambda \\
& =\hat{w}\left(s ; t+\left[\zeta^{-1}\right] ; \zeta\right) \hat{\tilde{w}}(s-1 ; t ; \zeta)-w_{0}\left(s ; t+\left[\zeta^{-1}\right]\right) \tilde{w}_{0}(s-1 ; t) .
\end{aligned}
$$

Using (2.42) and (2.43), and putting $\zeta=\lambda$, we obtain

$$
\frac{\hat{\tilde{w}}(s-1 ; t ; \lambda)}{\hat{\tilde{w}}(s ; t ; \lambda)}=\frac{w_{0}\left(s ; t+\left[\lambda^{-1}\right]\right)}{w_{0}(s-1 ; t)}
$$

Therefore, we have from definition (2.46),

$$
\frac{\partial \log \tau_{0}(s ; t)}{\partial t_{n}}-\frac{\partial \log \tau_{0}(s-1 ; t)}{\partial t_{n}}=-\frac{\partial \log w_{0}(s-1 ; t)}{\partial t_{n}} .
$$

Hence, we can fix the dependence of $\tau_{0}(s ; t)$ on $s$ by

$$
\log \tau_{0}(s ; t)-\log \tau_{0}(s-1 ; t)=-\log w_{0}(s-1 ; t) .
$$

Comparing this with the definition (2.45), we find that $\tau_{1}(s ; t)=\tau_{0}(s+1 ; t)$, as desired.

Statement (iii) is a direct consequence of (i) and the bilinear residue identity for the wave functions, (2.28).

\subsection{Construction of $\tau$ function}

In this subsection we construct $\tau$ functions of the cmKP hierarchy in terms of the free fermions or, in other words, the Clifford algebra as in the case of the KP hierarchy [3] or of the Toda lattice hierarchy $[15,16]$.

Let $\psi_{n}$ and $\psi_{n}^{*}(n \in \mathbb{Z})$ be free fermion operators, i.e., generators of a Clifford algebra $\mathcal{A}$ which satisfy the canonical anti-commutation relations:

$$
\left[\psi_{m}, \psi_{n}\right]_{+}=\left[\psi_{m}^{*}, \psi_{n}^{*}\right]_{+}=0, \quad\left[\psi_{m}, \psi_{n}^{*}\right]_{+}=\delta_{m n}
$$


where $[A, B]_{+}:=A B+B A$. The Fock space $\mathcal{F}$ and the dual Fock space $\mathcal{F}^{\vee}$ are generated by the vacuum vector $\mid$ vac $\rangle$ and its dual $\langle$ vac $|$ over $\mathcal{A}$ respectively. $\mathcal{F}$ and $\mathcal{F}^{\vee}$ contain states of charge $k,|k\rangle$ and $\langle k|$ respectively, which are characterized by

$$
\begin{aligned}
& \psi_{n}|k\rangle=\left\{\begin{array}{ll}
0, & n<k, \\
|k+1\rangle, & n=k,
\end{array} \quad \psi_{m}^{*}|k\rangle= \begin{cases}0, & m \geqq k, \\
|k-1\rangle, & m=k-1,\end{cases} \right. \\
& \langle k| \psi_{n}=\left\{\begin{array}{ll}
0, & n \geqq k, \\
\langle k-1|, & n=k-1,
\end{array} \quad\langle k| \psi_{m}^{*}= \begin{cases}0, & m<k, \\
\langle k+1|, & m=k .\end{cases} \right.
\end{aligned}
$$

In fact, $|\mathrm{vac}\rangle=|0\rangle$ and $\langle\mathrm{vac}|=\langle 0|$. The pairing of $\mathcal{F}$ and $\mathcal{F}^{\vee}$ is naturally defined by $\langle k \mid k\rangle=1$.

We define the operators $J(t), \psi(\lambda)$ and $\psi^{*}(\lambda)$ as follows:

$$
\begin{aligned}
J(t) & :=\sum_{n=1}^{\infty} t_{n} \sum_{k \in \mathbb{Z}} \psi_{k} \psi_{k+n}^{*}, \\
\psi(\lambda) & :=\sum_{n \in \mathbb{Z}} \psi_{n} \lambda^{n}, \quad \psi^{*}(\lambda):=\sum_{n \in \mathbb{Z}} \psi_{n}^{*} \lambda^{-n} .
\end{aligned}
$$

We quote important formulae from $\S 2.6$ of [3]:

$$
\begin{aligned}
& \langle m| e^{J(t)} \psi(\lambda)=\lambda^{m-1} e^{\xi(t, \lambda)}\langle m-1| e^{J\left(t-\left[\lambda^{-1}\right]\right)}, \\
& \langle m| e^{J(t)} \psi^{*}(\lambda)=\lambda^{-m} e^{-\xi(t, \lambda)}\langle m+1| e^{J\left(t+\left[\lambda^{-1}\right]\right)} .
\end{aligned}
$$

The bilinear identity comes from the following intertwining relation $[3, \S 2.1]$ :

$$
\sum_{n \in \mathbb{Z}} \psi_{n} g \otimes \psi_{n}^{*} g=\sum_{n \in \mathbb{Z}} g \psi_{n} \otimes g \psi_{n}^{*}
$$

where $g$ is an arbitrary element of the Clifford group generated by $\psi_{n}$ 's and $\psi_{n}^{*}$ 's.

Putting this equation between $\langle m+1| e^{J(t)} \otimes\left\langle m^{\prime}-1\right| e^{J\left(t^{\prime}\right)}$ and $|m\rangle \otimes\left|m^{\prime}\right\rangle$, we have

$$
\begin{aligned}
\operatorname{Res}_{\lambda} & =\infty\left\langle m+1\left|e^{J(t)} \psi(\lambda) g\right| m\right\rangle\left\langle m^{\prime}-1\left|e^{J\left(t^{\prime}\right)} \psi^{*}(\lambda) g\right| m^{\prime}\right\rangle \frac{d \lambda}{\lambda} \\
& =\sum_{n \in \mathbb{Z}}\left\langle m+1\left|e^{J(t)} \psi_{n} g\right| m\right\rangle\left\langle m^{\prime}-1\left|e^{J\left(t^{\prime}\right)} \psi_{n}^{*} g\right| m^{\prime}\right\rangle \\
& =\sum_{n \in \mathbb{Z}}\left\langle m+1\left|e^{J(t)} g \psi_{n}\right| m\right\rangle\left\langle m^{\prime}-1\left|e^{J\left(t^{\prime}\right)} g \psi_{n}^{*}\right| m^{\prime}\right\rangle .
\end{aligned}
$$

Thus we obtain

$$
\begin{aligned}
\operatorname{Res}_{\lambda}=\infty & \left\langle m+1\left|e^{J(t)} \psi(\lambda) g\right| m\right\rangle\left\langle m^{\prime}-1\left|e^{J\left(t^{\prime}\right)} \psi^{*}(\lambda) g\right| m^{\prime}\right\rangle \frac{d \lambda}{\lambda} \\
& = \begin{cases}0, & m^{\prime} \leqq m, \\
\left\langle m+1\left|e^{J(t)} g\right| m+1\right\rangle\left\langle m\left|e^{J\left(t^{\prime}\right)} g\right| m\right\rangle, & m^{\prime}=m+1\end{cases}
\end{aligned}
$$

due to (2.48).

For a Clifford group element $g$ we define $\tau$ functions by

$$
\tau_{0}(s ; t):=\left\langle n_{s}\left|e^{J(t)} g\right| n_{s}\right\rangle, \quad \tau_{1}(s ; t):=\left\langle n_{s}+1\left|e^{J(t)} g\right| n_{s}+1\right\rangle .
$$

The bilinear residue identity (2.41) holds because of (2.52), (2.53) and (2.55). Namely, we have constructed a pair of $\tau$ functions of the cmKP hierarchy for each $g$ in the Clifford group.

The action of $G L(\infty)$ mentioned at the end of Section 2.2 is realized as the action of the Clifford group, $g \mapsto g^{\prime} g\left(g^{\prime} \in G L(\infty)\right)$ in (2.56). Hence the above construction exhausts all the solutions of the cmKP hierarchy.

The vertex operator description of the $g l(\infty)$ symmetry is the same as that for the KP hierarchy in [3]. 


\section{Dispersionless modified KP hierarchy}

\subsection{Definition of the dcmKP hierarchy}

Let $N$ be a positive integer. When $n_{s}=N s$, we can introduce the parameter $\hbar$ into the cmKP hierarchy and take the dispersionless limit, as is done for the mKP hierarchy in [17]. Now for the dcmKP hierarchy, the independent variables are the continuous variables $s, x$ and $t=\left(t_{1}, t_{2}, \ldots\right)$. The dependent variables are encapsulated in $\mathcal{L}(k ; s, t)$ and $\mathcal{P}(k ; s, t)$, which are respectively formal power series and polynomial of $k$ having the following form:

$$
\begin{aligned}
& \mathcal{L}(s)=\mathcal{L}(k ; s, t)=k+u_{1}(s, t)+u_{2}(s, t) k^{-1}+\cdots=\sum_{n=0}^{\infty} u_{n}(s, t) k^{1-n}, \\
& \mathcal{P}(s)=\mathcal{P}(k ; s, t)=p_{0}(s, t) k^{N}+\cdots+p_{N-1}(s, t) k=\sum_{n=0}^{N-1} p_{n}(s, t) k^{N-n} .
\end{aligned}
$$

Here $u_{0}=1, p_{0} \neq 0$. We do not write the dependence on $x$ explicitly for the reason we are going to see later.

The $N$-dcmKP hierarchy is the following system of equations:

$$
\begin{aligned}
& \frac{\partial \mathcal{L}}{\partial t_{n}}=\left\{\mathcal{B}_{n}, \mathcal{L}\right\}, \quad \mathcal{B}_{n}:=\left(\mathcal{L}^{n}\right)_{>0}, \\
& \frac{\partial \mathcal{L}}{\partial s}=\{\log \mathcal{P}, \mathcal{L}\}, \\
& \frac{\partial \log \mathcal{P}}{\partial t_{n}}=\frac{\partial \mathcal{B}_{n}}{\partial s}-\left\{\log \mathcal{P}, \mathcal{B}_{n}\right\}
\end{aligned}
$$

where now the projection $(\cdot)_{>0}$ is with respect to $k, \log \mathcal{P}$ is formally understood as

$$
\log \mathcal{P}=\log p_{0}+\log k^{N}+\sum_{n=1}^{\infty} \mathfrak{p}_{n} k^{-n},
$$

and $\{\cdot, \cdot\}$ is the Poisson bracket

$$
\{f(k, x), g(k, x)\}=\frac{\partial f}{\partial k} \frac{\partial g}{\partial x}-\frac{\partial f}{\partial x} \frac{\partial g}{\partial k} .
$$

As usual, the $n=1$ case of equations (3.3) and (3.5) implies that the dependence on $x$ and $t_{1}$ appears in the combination $x+t_{1}$. As a result, we usually omit $x$ and identify $t_{1}$ with $x$.

By the standard argument, equation (3.3) is equivalent to the Zakharov-Shabat (or zerocurvature) equations

$$
\frac{\partial \mathcal{B}_{n}}{\partial t_{m}}-\frac{\partial \mathcal{B}_{m}}{\partial t_{n}}+\left\{\mathcal{B}_{n}, \mathcal{B}_{m}\right\}=0, \quad \text { or } \quad \frac{\partial \mathcal{B}_{n}^{c}}{\partial t_{m}}-\frac{\partial \mathcal{B}_{m}^{c}}{\partial t_{n}}+\left\{\mathcal{B}_{n}^{c}, \mathcal{B}_{m}^{c}\right\}=0
$$

where $\mathcal{B}_{n}^{c}=-\left(\mathcal{L}^{n}\right)_{\leq 0}=\mathcal{B}_{n}-\mathcal{L}^{n}$.

Remark 2. As in the case of the cmKP hierarchy (cf. Remark 1), even when we start from polynomial $\mathcal{P}$ with a constant term, $\mathcal{P}(s)=p_{0}(s) k^{N}+\cdots+p_{N}(s)$, we can gauge away $p_{N}(s)$. See Appendix A.

In general, we can let $\mathcal{P}$ be a power series with leading term $p_{0} k^{N}$, and have infinitely many negative power terms, i.e., $\mathcal{P}=\sum_{n=0}^{\infty} p_{n} k^{N-n}$. In particular, if $\mathcal{P}=\mathcal{L}^{N}$, then equation (3.4) says that there is no dependence on $s$, and (3.5) is equivalent to (3.3). This is the dmKP hierarchy considered by Kupershmidt [8], Chang and Tu [1]. 
Remark 3. Suppose $(\mathcal{L}, \mathcal{P})$ is a solution of N-dcmKP hierarchy $(3.3)-(3.5)$ and $Q(\zeta)=a_{m} \zeta^{m}+$ $\cdots+a_{0}$ is a polynomial with coefficients $a_{0}, \ldots, a_{m}$ independent of $s, x$ and $t$. If $Q(\mathcal{L}(k)) \mathcal{P}(k)$ is a polynomial of $k$ without constant term ${ }^{1}$, then it is easy to see that $(\mathcal{L}, \mathcal{P} Q(\mathcal{L}))$ is a solution of $(N+m)$-dcmKP hierarchy. We say that this solution is equivalent to the solution $(\mathcal{L}, \mathcal{P})$.

\subsection{Dressing operator}

As in [21], we can show the existence of a dressing operator.

Proposition 4. For any solution $(\mathcal{L}(s), \mathcal{P}(s))$ of the dcmKP hierarchy, there exists an operator $\exp \operatorname{ad} \phi(s)$ that satisfies

$$
\begin{aligned}
& \mathcal{L}=(\exp \operatorname{ad} \phi) k, \\
& \nabla_{t_{n}, \phi} \phi=\mathcal{B}_{n}^{c}, \quad \nabla_{s, \phi} \phi=\log \mathcal{P}-\log \mathcal{L}^{N},
\end{aligned}
$$

where $\phi(s)$ is a power series of the form $\phi(s)=\sum_{n=0}^{\infty} \phi_{n}(s ; t) k^{-n},(\operatorname{ad} f) g=\{f, g\}$ and

$$
\nabla_{u, \psi} \phi=\sum_{m=0}^{\infty} \frac{1}{(m+1) !}(\operatorname{ad} \psi)^{m} \frac{\partial \phi}{\partial u}
$$

for series $\psi, \phi$ and variable $u$.

Comparing the coefficients of $k^{0}$ on both sides of equations in (3.7), we have

Corollary 3. The function $\phi_{0}(s, t)$ satisfies

$$
\frac{\partial \phi_{0}}{\partial t_{n}}=-\left(\mathcal{L}^{n}\right)_{0}, \quad \frac{\partial \phi_{0}}{\partial s}=\log p_{0} .
$$

\subsection{Orlov-Schulman function}

Using the dressing operator $\phi$, we can construct the Orlov-Schulman function $\mathcal{M}$ by

$$
\begin{aligned}
\mathcal{M} & =e^{\operatorname{ad} \phi}\left(\sum_{n=1}^{\infty} n t_{n} k^{n-1}+x+N s k^{-1}\right) \\
& =\sum_{n=1}^{\infty} n t_{n} \mathcal{L}^{n-1}+x+\frac{N s}{\mathcal{L}}+\sum_{n=1}^{\infty} v_{n} \mathcal{L}^{-n-1},
\end{aligned}
$$

where $v_{n}$ are functions of $s, t$. $\mathcal{M}$ has the property that it forms a canonical pair with $\mathcal{L}$, namely

$$
\{\mathcal{L}, \mathcal{M}\}=1
$$

Using Lemma A.1 in Appendix A of [21] and Proposition 4, we find that

$$
\frac{\partial \mathcal{M}}{\partial t_{n}}=\left\{\mathcal{B}_{n}, \mathcal{M}\right\}, \quad \frac{\partial \mathcal{M}}{\partial s}=\{\log \mathcal{P}, \mathcal{M}\}
$$

As in [20], we can show by using the equations (3.3)-(3.5), (3.10), (3.9) and Corollary 3 that the expansion of $\mathcal{B}_{n}$ and $\log \mathcal{P}$ with respect to $\mathcal{L}$ can be expressed through the functions $v_{n}$. More precisely,

\footnotetext{
${ }^{1}$ We do not need to impose this condition when we consider the generalized cmKP hierarchy as in Remark 2.
} 
Proposition 5. We have the following relations:

$$
\begin{aligned}
& \mathcal{B}_{n}=\mathcal{L}^{n}+\frac{\partial \phi_{0}}{\partial t_{n}}-\sum_{m=1}^{\infty} \frac{1}{m} \frac{\partial v_{m}}{\partial t_{n}} \mathcal{L}^{-m} \\
& \log \mathcal{P}=\log \mathcal{L}^{N}+\frac{\partial \phi_{0}}{\partial s}-\sum_{m=1}^{\infty} \frac{1}{m} \frac{\partial v_{m}}{\partial s} \mathcal{L}^{-m} .
\end{aligned}
$$

\subsection{Fundamental two form and $S$ function}

The fundamental two form $\omega$ is defined by

$$
\omega=d k \wedge d x+\sum_{n=1}^{\infty} d \mathcal{B}_{n} \wedge d t_{n}+d \log \mathcal{P} \wedge d s .
$$

The exterior derivative $d$ is taken with respect to the independent variables $k, x, s$ and $t$. From definition, $\omega$ is closed

$$
d \omega=0,
$$

and it follows from the zero-curvature equation (3.6) and equation (3.5) that

$$
\omega \wedge \omega=0 .
$$

$(\mathcal{L}, \mathcal{M})$ is a pair of functions that play the role of Darboux coordinates. Namely

$$
d \mathcal{L} \wedge d \mathcal{M}=\omega .
$$

In fact, we can prove as Proposition 2 in [20] that

Proposition 6. The system of equations (3.3)-(3.5), (3.10) and (3.9) are equivalent to

$$
d \mathcal{L} \wedge d \mathcal{M}=d k \wedge d x+\sum_{n=1}^{\infty} d \mathcal{B}_{n} \wedge d t_{n}+d \log \mathcal{P} \wedge d s
$$

This formula implies that there exists a function $S(\mathcal{L} ; s, t)$ such that

$$
d S=\mathcal{M} d \mathcal{L}+k d x+\sum_{n=1}^{\infty} \mathcal{B}_{n} d t_{n}+\log \mathcal{P} d s
$$

or equivalently,

$$
\frac{\partial S}{\partial \mathcal{L}}=\mathcal{M}, \quad \frac{\partial S}{\partial x}=\frac{\partial S}{\partial t_{1}}=k, \quad \frac{\partial S}{\partial t_{n}}=\mathcal{B}_{n}, \quad \frac{\partial S}{\partial s}=\log \mathcal{P} .
$$

From the formula (3.8) and Proposition 5, it is easy to see that

Proposition 7. The S function is given explicitly by

$$
S=\sum_{n=1}^{\infty} t_{n} \mathcal{L}^{n}+x \mathcal{L}+s \log \mathcal{L}^{N}-\sum_{n=1}^{\infty} \frac{v_{n}}{n} \mathcal{L}^{-n}+\phi_{0} .
$$




\subsection{Tau function}

We introduce the power series $\mathrm{k}(z ; s, t)$ as the (formal) inverse of $\mathcal{L}(k ; s, t)$ with respect to $k$, i.e. $\mathcal{L}(\mathrm{k}(z ; s, t) ; s, t)=z$ and $\mathrm{k}(\mathcal{L}(k ; s, t) ; s, t)=k$. Define the Grunsky coefficients $b_{m n}, m, n>0$ and $b_{n 0}=b_{0 n}, n>0$ of $\mathrm{k}(z)=\mathrm{k}(z ; s, t)$ (cf. [4, 9, 19]) by the expansions

$$
\begin{aligned}
& \log \frac{\mathrm{k}\left(z_{1}\right)-\mathrm{k}\left(z_{2}\right)}{z_{1}-z_{2}}=-\sum_{m=1}^{\infty} b_{m n} z_{1}^{-m} z_{2}^{-n} \\
& \log \frac{\mathrm{k}(z)}{z}=-\sum_{n=1}^{\infty} b_{n 0} z^{-n}
\end{aligned}
$$

Obviously, $b_{m n}$ are symmetric. In terms of the Grunsky coefficients, we have (cf. $\left.[4,9,19]\right)$

$$
\left(\mathcal{L}^{n}\right)_{0}=n b_{n, 0}, \quad \mathcal{B}_{n}=\mathcal{L}^{n}-n b_{n, 0}+n \sum_{m=1}^{\infty} b_{n m} \mathcal{L}^{-m}
$$

Comparing with (3.11), we find that

$$
\frac{\partial v_{m}}{\partial t_{n}}=-n m b_{n m}, \quad \frac{\partial \phi_{0}}{\partial t_{n}}=-n b_{n, 0} .
$$

Therefore by the symmetry of Grunsky coefficients, the first equation gives

$$
\frac{\partial v_{m}}{\partial t_{n}}=\frac{\partial v_{n}}{\partial t_{m}} .
$$

Consequently, we have

Proposition 8. There exists a tau function $\tau_{\mathrm{dcmKP}}(s ; t)$, determined up to a function of $s$, such that

$$
\frac{\partial \log \tau_{\mathrm{dcmKP}}}{\partial t_{n}}=v_{n} .
$$

Define $\mathcal{F}=\log \tau_{\text {dcmKP. }}$. It is called the free energy. Using equations (3.19) and (3.17), we can rewrite the equations (3.14), (3.15) as

$$
\begin{aligned}
& z_{1} \exp \left(\sum_{n=1}^{\infty} \frac{1}{n} \frac{\partial \phi_{0}}{\partial t_{n}} z_{1}^{-n}\right)-z_{2} \exp \left(\sum_{n=1}^{\infty} \frac{1}{n} \frac{\partial \phi_{0}}{\partial t_{n}} z_{2}^{-n}\right) \\
& =\left(z_{1}-z_{2}\right) \exp \left(\sum_{m=1}^{\infty} \sum_{n=1}^{\infty} \frac{1}{m n} \frac{\partial^{2} \mathcal{F}}{\partial t_{m} \partial t_{n}} z_{1}^{-m} z_{2}^{-n}\right) .
\end{aligned}
$$

Comparing the coefficients of $z_{2}^{0}$ on both sides, we have

$$
z \exp \left(\sum_{n=1}^{\infty} \frac{1}{n} \frac{\partial \phi_{0}}{\partial t_{n}} z^{-n}\right)=z+\frac{\partial \phi_{0}}{\partial t_{1}}-\sum_{n=1}^{\infty} \frac{1}{n} \frac{\partial^{2} \mathcal{F}}{\partial t_{n} \partial t_{1}} z^{-n} .
$$

On the other hand, we can formulate a partial converse of Proposition 8 as:

Proposition 9. If $\tau_{\mathrm{dcmKP}}(s, t)$ and $\phi_{0}(s, t)$ are functions that satisfy the equation (3.20), then the pair of functions $(\mathcal{L}, \mathcal{P})$, where $\mathcal{L}(k)=\mathcal{L}(k ; s, t)$ is defined by taking the inverse of the formal power series

$$
\mathrm{k}(z)=\mathrm{k}(z ; s, t)=z \exp \left(\sum_{n=1}^{\infty} \frac{1}{n} \frac{\partial \phi_{0}}{\partial t_{n}} z^{-n}\right)
$$


and $\mathcal{P}(k)=\mathcal{P}(k ; s, t)$ is defined so that its composition with $\mathrm{k}(z)$ is given by

$$
\mathcal{P}(\mathrm{k}(z))=\mathcal{P}(\mathrm{k}(z ; s, t) ; s, t)=\exp \left(\frac{\partial \phi_{0}}{\partial s}\right) z^{N} \exp \left(-\sum_{n=1}^{\infty} \frac{1}{n} \frac{\partial^{2} \log \tau_{\mathrm{dcmKP}}}{\partial s \partial t_{n}} z^{-n}\right),
$$

satisfy the dcmKP hierarchy (3.3)-(3.5), in the generalized sense as Remark 2.

Proof. From (3.21), we have

$$
\mathrm{k}(z)=z+\frac{\partial \phi_{0}}{\partial t_{1}}-\sum_{n=1}^{\infty} \frac{1}{n} \frac{\partial^{2} \mathcal{F}}{\partial t_{n} \partial t_{1}} z^{-n}
$$

Therefore, it follows immediately from the definition of $\log \mathcal{P}$ that

$$
\left.\frac{\partial \log \mathcal{P}}{\partial t_{1}}\right|_{\mathcal{L} \text { fixed }}=\left.\frac{\partial \mathrm{k}(\mathcal{L})}{\partial s}\right|_{\mathcal{L} \text { fixed }} .
$$

As in the proof of Proposition 3.2 in [19], this implies equation (3.4).

On the other hand, let $b_{m n}, m, n \geq 0$ be the Grunsky coefficients of $\mathrm{k}(z)$ defined as in (3.14), (3.15). Comparing the equations (3.20) with (3.14), (3.15), we find from (3.16) that

$$
\mathcal{B}_{n}=\mathcal{L}^{n}+\frac{\partial \phi_{0}}{\partial t_{n}}-\sum_{n=1}^{\infty} \frac{1}{m} \frac{\partial^{2} \log \tau_{\mathrm{dcmKP}}}{\partial t_{m} \partial t_{n}} \mathcal{L}^{-m}
$$

Therefore,

$$
\left.\frac{\partial \mathcal{B}_{n}}{\partial t_{1}}\right|_{\mathcal{L} \text { fixed }}=\left.\frac{\partial \mathrm{k}(\mathcal{L})}{\partial t_{n}}\right|_{\mathcal{L} \text { fixed }},\left.\quad \frac{\partial \mathcal{B}_{n}}{\partial s}\right|_{\mathcal{L} \text { fixed }}=\left.\frac{\partial \log \mathcal{P}}{\partial t_{n}}\right|_{\mathcal{L} \text { fixed }} .
$$

The first equation implies equation (3.3). On the other hand, by using the second equation in (3.23) and equations (3.3) and (3.4), we have

$$
\begin{aligned}
& \frac{\partial \log \mathcal{P}}{\partial t_{n}}-\frac{\partial \mathcal{B}_{n}}{\partial s}+\left\{\log \mathcal{P}, \mathcal{B}_{n}\right\} \\
& \quad=\left.\frac{\partial \log \mathcal{P}}{\partial t_{n}}\right|_{\mathcal{L} \text { fixed }}+\frac{\partial \log \mathcal{P}}{\partial \mathcal{L}} \frac{\partial \mathcal{L}}{\partial t_{n}}-\left.\frac{\partial \mathcal{B}_{n}}{\partial s}\right|_{\mathcal{L} \text { fixed }}-\frac{\partial \mathcal{B}_{n}}{\partial \mathcal{L}} \frac{\partial \mathcal{L}}{\partial s} \\
& \quad+\left.\frac{\partial \log \mathcal{P}}{\partial \mathcal{L}} \frac{\partial \mathcal{L}}{\partial k} \frac{\partial \mathcal{B}_{n}}{\partial t_{1}}\right|_{\mathcal{L} \text { fixed }}-\left.\frac{\partial \log \mathcal{P}}{\partial t_{1}}\right|_{\mathcal{L} \text { fixed }} \frac{\partial \mathcal{B}_{n}}{\partial \mathcal{L}} \frac{\partial \mathcal{L}}{\partial k}=0 .
\end{aligned}
$$

This gives equation (3.5).

\subsection{Relation with the dmKP hierarchy}

The dmKP hierarchy in [17] is defined by the system of equations

$$
\begin{aligned}
& \frac{\partial \mathcal{L}^{\mathrm{dmKP}}}{\partial t_{n}}=\left\{\mathcal{B}_{n}^{\mathrm{dmKP}}, \mathcal{L}^{\mathrm{dmKP}}\right\}, \\
& \frac{\partial \mathcal{L}^{\mathrm{dmKP}}}{\partial s}=\left\{\log \mathcal{P}^{\mathrm{dmKP}}, \mathcal{L}^{\mathrm{dmKP}}\right\}, \\
& \frac{\partial \log \mathcal{P}^{\mathrm{dmKP}}}{\partial t_{n}}=\frac{\partial \mathcal{B}_{n}^{\mathrm{dmKP}}}{\partial s}-\left\{\log \mathcal{P}^{\mathrm{dmKP}}, \mathcal{B}_{n}^{\mathrm{dmKP}}\right\},
\end{aligned}
$$


for the power series

$$
\begin{aligned}
& \mathcal{L}^{\mathrm{dmKP}}(k ; s, t)=k+u_{2}^{\mathrm{dmKP}}(s, t) k^{-1}+u_{3}^{\mathrm{dmKP}} k^{-2}+\cdots, \\
& \mathcal{B}_{n}^{\operatorname{dmKP}}(k ; s, t)=\left(\mathcal{L}^{\mathrm{dmKP}}(k ; s, t)^{n}\right)_{\geq 0}, \\
& \mathcal{P}^{\operatorname{dmKP}}(k ; s, t)=k^{N}+q_{1}(s, t) k^{N-1}+\cdots+q_{N}(s, t) .
\end{aligned}
$$

We have

Proposition 10. If $(\mathcal{L}(s), \mathcal{P}(s))$ is a solution of the dcmKP hierarchy, then the pair $\left(\mathcal{L}^{\mathrm{dcmKP}}(s)\right.$, $\left.\mathcal{P}^{\mathrm{dcmKP}}(s)\right)$, where

$$
\begin{aligned}
& \mathcal{L}^{\operatorname{dmKP}}(s)=\mathcal{L}^{\operatorname{dmKP}}(k ; s, t)=\mathcal{L}\left(k-u_{1}(s, t) ; s, t\right), \\
& \mathcal{P}^{\operatorname{dmKP}}(s)=\mathcal{P}^{\operatorname{dmKP}}(k ; s, t)=p_{0}(s, t)^{-1} \mathcal{P}\left(k-u_{1}(s, t) ; s, t\right),
\end{aligned}
$$

is a solution of the dmKP hierarchy.

Proof. It is easy to see that $\mathcal{L}^{\mathrm{dmKP}}$ and $\mathcal{P}^{\mathrm{dmKP}}$ defined by (3.29) has the form required by (3.27) and (3.28). Let $\exp \left(\operatorname{ad} \sum_{n=0}^{\infty} \phi_{n}(s, t) k^{-n}\right)$ be the dressing operator of the solution $(\mathcal{L}(s), \mathcal{P}(s))$. Using Corollary 3 , it is easy to check that $\mathcal{L}^{\mathrm{dmKP}}, \log \mathcal{P}^{\mathrm{dmKP}}$ can be written as

$$
\mathcal{L}^{\mathrm{dmKP}}=e^{-\mathrm{ad} \phi_{0}} \mathcal{L}, \quad \log \mathcal{P}^{\mathrm{dmKP}}=e^{-\operatorname{ad} \phi_{0}} \log \mathcal{P}-\frac{\partial \phi_{0}}{\partial s} .
$$

Since

$$
e^{-\operatorname{ad} \phi_{0}}\left(\mathcal{L}^{n}\right)_{>0}+\left(\mathcal{L}^{n}\right)_{0}=e^{-\operatorname{ad} \phi_{0}}\left(\mathcal{L}^{n}\right)_{\geq 0}=\left(e^{-\operatorname{ad} \phi_{0}} \mathcal{L}^{n}\right)_{\geq 0}
$$

we have

$$
\mathcal{B}_{n}^{\mathrm{dmKP}}=e^{-\operatorname{ad} \phi_{0}} \mathcal{B}_{n}-\frac{\partial \phi_{0}}{\partial t_{n}}
$$

Using this relation, equation (3.30), equations (3.3)-(3.5) and Lemma A.1 in [21], it is a direct computation to verify that $\left(\mathcal{L}^{\mathrm{dmKP}}, \mathcal{P}^{\mathrm{dmKP}}\right)$ satisfy equations $(3.24)-(3.26)$.

The map (3.29) is called a dispersionless Miura map, corresponding to the Miura map between a solution of KdV hierarchy and a solution of modified KdV hierarchy.

\subsection{The special case $\mathcal{P}=p_{0} k$}

In the special case where $N=1$ and $\mathcal{P}=p_{0} k$, we have from Corollary 3 and equation (3.15), (3.17),

$$
\log \mathcal{P}=\log p_{0}+\log k=\frac{\partial \phi_{0}}{\partial s}+\log \mathcal{L}+\sum_{n=1}^{\infty} \frac{\partial \phi_{0}}{\partial t_{n}} \mathcal{L}^{-n} .
$$

Comparing with (3.12), we find that

$$
\frac{\partial^{2} \log \tau_{\mathrm{dcmKP}}}{\partial t_{n} \partial s}=\frac{\partial v_{n}}{\partial s}=-\frac{\partial \phi_{0}}{\partial t_{n}} .
$$

Therefore we can fix the dependence of $\tau_{\mathrm{dcmKP}}$ on $s$ by the equation

$$
\frac{\partial \log \tau_{\mathrm{dcmKP}}}{\partial s}=-\phi_{0}
$$


and equation (3.20) can be written as

$$
\begin{gathered}
z_{1} \exp \left(-\sum_{n=1}^{\infty} \frac{1}{n} \frac{\partial^{2} \mathcal{F}}{\partial s \partial t_{n}} z_{1}^{-n}\right)-z_{2} \exp \left(-\sum_{n=1}^{\infty} \frac{1}{n} \frac{\partial^{2} \mathcal{F}}{\partial s \partial t_{n}} z_{2}^{-n}\right) \\
=\left(z_{1}-z_{2}\right) \exp \left(\sum_{m=1}^{\infty} \sum_{n=1}^{\infty} \frac{1}{m n} \frac{\partial^{2} \mathcal{F}}{\partial t_{m} \partial t_{n}} z_{1}^{-m} z_{2}^{-n}\right),
\end{gathered}
$$

which we call the dispersionless Hirota equation for dcmKP hierarchy with $\mathcal{P}=p_{0} k$. The counterpart of Proposition 9 becomes

Proposition 11. If $\tau_{\mathrm{dcmKP}}(s, t)$ is a function that satisfies the dispersionless Hirota equation (3.32), then the pair of functions $(\mathcal{L}, \mathcal{P})$, where $\mathcal{L}(k)=\mathcal{L}(k ; s, t)$ is defined by inverting

$$
\mathrm{k}(z)=\mathrm{k}(z ; s, t)=z \exp \left(-\sum_{n=1}^{\infty} \frac{1}{n} \frac{\partial^{2} \log \tau_{\mathrm{dcmKP}}}{\partial t_{n} \partial s} z^{-n}\right)
$$

and

$$
\mathcal{P}(k)=\mathcal{P}(k ; s, t)=k \exp \left(-\frac{\partial^{2} \log \tau_{\mathrm{dcmKP}}}{\partial s^{2}}\right)
$$

satisfy the dcmKP hierarchy (3.3)-(3.5).

Proof. This can be directly deduced from Proposition 9.

Comparing with Proposition 3.1 in [19] and its following discussion, we find that if $(\mathcal{L}(k ; s, t)$, $\left.\mathcal{P}(k ; s, t)=p_{0}(s, t) k\right)$ is a solution of dcmKP hierarchy, $\mathcal{L}(k ; s, t)$ is a solution of the hierarchy

$$
\frac{\partial \mathcal{L}}{\partial t_{n}}=\left\{\left(\mathcal{L}^{n}\right)_{\geq 0}, \mathcal{L}\right\}_{T}
$$

$\mathcal{L}_{1}(k ; s, t)=\mathcal{L}\left(p_{0}^{-1} k ; s, t\right)$ is a solution of the hierarchy

$$
\frac{\partial \mathcal{L}_{1}}{\partial t_{n}}=\left\{\left(\mathcal{L}_{1}^{n}\right)_{>0}, \mathcal{L}_{1}\right\}_{T}
$$

and $\mathcal{L}_{1 / 2}(k ; s, t)=\mathcal{L}\left(p_{0}^{-1 / 2} k ; s, t\right)$ is a solution of the hierarchy

$$
\frac{\partial \mathcal{L}_{1 / 2}}{\partial t_{n}}=\left\{\left(\mathcal{L}_{1 / 2}^{n}\right)_{>0}+\frac{1}{2}\left(\mathcal{L}_{1 / 2}^{n}\right)_{0}, \mathcal{L}_{1 / 2}\right\}_{T}
$$

Here $\{\cdot, \cdot\}$ is the Poisson bracket of dToda hierarchy:

$$
\{f(k, s), g(k, s)\}_{T}=k \frac{\partial f}{\partial k} \frac{\partial g}{\partial s}-k \frac{\partial f}{\partial s} \frac{\partial g}{\partial k} .
$$

(3.33) and (3.34) can be considered as gauge equivalent form of the dcmKP hierarchy with $\mathcal{P}=p_{0} k$ and with gauge parameter 1 and $1 / 2$ respectively. For the cmKP version of (3.33) and (3.34) we refer to Appendices B, C and D. The form (3.34) was used in the work [22]. 


\subsection{Quasi-classical limit of the cmKP hierarchy}

The dispersionless KP hierarchy and the dispersionless Toda hierarchy are obtained as quasiclassical limit of corresponding "dispersionful" hierarchies. See [21]. The dcmKP hierarchy is also quasi-classical limit of the cmKP hierarchy. In this subsection we briefly explain the correspondence.

Let us define the order as in [21], § 1.7.1:

$$
\operatorname{ord}^{\hbar}\left(\sum a_{n, m}(t) \hbar^{n} \partial^{m}\right):=\max \left\{m-n \mid a_{n, m}(t) \neq 0\right\} .
$$

In particular, $\operatorname{ord}^{\hbar}(\hbar)=-1, \operatorname{ord}^{\hbar}(\partial)=1$. The principal symbol (resp. the symbol of order $l$ ) of an operator $A=\sum a_{n, m} \hbar^{n} \partial^{m}$ is

$$
\sigma^{\hbar}(A):=\hbar^{-\operatorname{ord}^{\hbar}(A)} \sum_{m-n=\operatorname{ord}^{\hbar}(A)} a_{n, m} k^{m}
$$

respectively

$$
\sigma_{l}^{\hbar}(A):=\hbar^{-l} \sum_{m-n=l} a_{n, m} k^{m} .
$$

Let us redefine the cmKP hierarchy with a small parameter $\hbar$ as follows. Fix a positive integer $N$. The discrete independent variable $s$ runs in $S:=\hbar \mathbb{Z}$. Operators $L, P$ are of the form

$$
\begin{aligned}
& L(s ; x, t)=\sum_{n=0}^{\infty} u_{n}(\hbar, s, x, t)(\hbar \partial)^{1-n}, \\
& P(s ; x, t)=\sum_{n=0}^{N-1} p_{n}(\hbar, s, x, t)(\hbar \partial)^{N-n},
\end{aligned}
$$

where $u_{0}=1, p_{0} \neq 0$ and all coefficients $u_{n}(\hbar, s, x, t)$ and $p_{n}(\hbar, s, x, t)$ are regular in $\hbar$. Namely they do not contain negative powers of $\hbar$. The cmKP hierarchy is rewritten as

$$
\begin{aligned}
& \hbar \frac{\partial L(s)}{\partial t_{n}}=\left[B_{n}(s), L(s)\right], \\
& (L(s+\hbar)-L(s)) P(s)=[P(s), L(s)], \\
& \left(B_{n}(s)-B_{n}(s+\hbar)\right) P(s)=\left[P(s), \hbar \frac{\partial}{\partial t_{n}}-B_{n}(s)\right] .
\end{aligned}
$$

where $B_{n}(s)=B_{n}(s ; x, t)$ is defined as before. The principal symbols of (3.38), (3.39) and (3.40) give equations $(3.3),(3.4)$ and (3.5) of the dcmKP hierarchy respectively, where $\mathcal{L}(s)=\sigma_{0}^{\hbar}(L(s))$ and $\mathcal{P}(s)=\sigma_{0}^{\hbar}(P(s))$.

The dressing operator $W(s ; x, t ; \partial)(2.9)$ should have the form

$$
\begin{aligned}
& W(s ; x, t ; \hbar ; \partial)=\exp \left(\hbar^{-1} X(\hbar, x, t ; \partial)\right)(\hbar \partial)^{N s}, \\
& X(\hbar, x, t ; \partial)=\sum_{n=0}^{\infty} \chi_{n}(\hbar, x, t)(\hbar \partial)^{-n}
\end{aligned}
$$

where $\chi_{n}(\hbar, x, t)$ is regular in $\hbar$. The principal symbol of $X$ is the function $\phi$ in Proposition 4 .

Remark 4. Solutions of the dispersionless KP and Toda hierarchies can be lifted up to solutions of the KP and Toda hierarchies (with $\hbar$ ) respectively by lifting the dressing operator. See Corollary 1.7.6 and Corollary 2.7.6 of [21]. 
As for the dcmKP hierarchy, we conjecture that any solution of the dcmKP hierarchy would be lifted up to a solution of the cmKP hierarchy but there is difficulty coming from the form of $P(s)$. Naive lift of $\phi(s, t)$ would cause a tail of $P(s)$ which has non-positive order as a microdifferential operator and negative order in the sense of (3.35). We can correct this by inductively modifying $X(s)$ to get $P(s)$ of the form (3.37), but this inductive procedure might make $X(s)$ behave wildly with respect to $s$.

In the context of the WKB analysis of the linear equations (2.12) the $S$ function introduced in Section 3.4 is the phase function:

$$
\begin{aligned}
& w(s ; x, t ; \lambda)=\exp \left(\hbar^{-1}(S+O(\hbar))\right), \\
& S=\sum_{n=1}^{\infty} t_{n} \lambda^{n}+x \lambda+N s \log \lambda-\sum_{n=1}^{\infty} \frac{v_{n}}{n} \lambda^{-n}+\phi_{0} .
\end{aligned}
$$

By replacing $\lambda$ with $\mathcal{L}$, we obtain the $S$ function in Proposition 7 .

If the conjecture in Remark 4 is true, the form of $\mathcal{P}(s)$ of the dcmKP hierarchy reduces drastically by the following proposition.

Proposition 12. If a solution $(\mathcal{L}(s), \mathcal{P}(s))_{s \in S}$ of the dcmKP hierarchy is the quasi-classical limit of a solution $(L(s), P(s))_{s \in S}$ of the cmKP hierarchy with $\hbar$. Then $\mathcal{P}(s)=p_{0}(s, t) k^{N}$.

Proof. Let $\tau_{0}(s ; t)$ and $\tau_{1}(s ; t)$ be the tau function of $(L(s), P(s))_{s \in S}$. They are expressed by the Clifford algebra as in (2.56): for $s \in \mathbb{Z} \hbar$

$$
\begin{aligned}
& \tau_{0}(\hbar ; s ; t)=\left\langle N s \hbar^{-1}\left|e^{J(t) \hbar^{-1}} g\right| N s \hbar^{-1}\right\rangle, \\
& \tau_{1}(\hbar ; s ; t)=\left\langle N s \hbar^{-1}+1\left|e^{J(t) \hbar^{-1}} g\right| N s \hbar^{-1}+1\right\rangle .
\end{aligned}
$$

Defining

$$
\tau(s ; t):=\left\langle s \hbar^{-1}\left|e^{J(t) \hbar^{-1}} g\right| s \hbar^{-1}\right\rangle
$$

we have a solution $(\tilde{L}(s), \tilde{P}(s))_{s \in \mathbb{Z}}$ of the cmKP hierarchy with $N=1$ whose $\tau$ functions are $\tilde{\tau}_{0}(s ; t)=\tau(s ; t), \tilde{\tau}_{1}(s ; t)=\tau(s+\hbar ; t)$. It is easy to see that the dressing operators $W(s)$ of $(L(s), P(s))_{s \in S}$ and $\tilde{W}(s)$ of $(\tilde{L}(s), \tilde{P}(s))_{s \in \mathbb{Z}}$ are related by $W(s)=\tilde{W}(N s)$.

Hence $L(s)=\tilde{L}(N s)$ and $P(s)=\tilde{P}(N s+(N-1) \hbar) \cdots \tilde{P}(N s)$. The symbol of order 0 of the last equation gives

$$
\mathcal{P}(s)=\sigma_{0}^{\hbar}(P(s))=\sigma_{0}^{\hbar}(\tilde{P}(N s+(N-1) \hbar)) \cdots \sigma_{0}^{\hbar}(\tilde{P}(N s)),
$$

because $\sigma_{0}^{\hbar}(A B)=\sigma_{0}^{\hbar}(A) \sigma_{0}^{\hbar}(B)$ for any operators $A, B$, $\operatorname{ord}^{\hbar}(A)=\operatorname{ord}^{\hbar}(B)=0$. Since $\tilde{P}(s)$ is of the form $\tilde{p}_{0}(s, t) \hbar \partial, \mathcal{P}(s)$ is of the form $p_{0}(s, t) k^{N}$.

A proof of the above statement without lifting up to the cmKP hierarchy is desirable.

If $\mathcal{P}(s)=p_{0}(s, t) k^{N}$, we have $\operatorname{Res} \mathcal{L}^{n} d_{k} \log \mathcal{P}(s)=N\left(\mathcal{L}^{n}\right)_{0}$, which is equivalent to

$$
\frac{\partial v_{n}}{\partial s}=-N \frac{\partial \phi_{0}}{\partial t_{n}}
$$

because of (3.12) and Corollary 3. This equation together with (3.18) is a compatibility condition of equations (3.19) and

$$
\frac{\partial \log \tau_{\mathrm{dcmKP}}}{\partial s}=-N \phi_{0},
$$

which fixes the $s$-dependence of $\log \tau_{\text {dcmKP. }}$. 
In fact, this is consistent with the quasi-classical limit. We express the $\tau$ functions of $(L(s), P(s))_{s \in S}$ as $(3.43)$ and define $\tau(s ; t)$ by (3.44). As in [21], that $\tau$ function behaves as

$$
\tau(s ; t)=e^{\hbar^{-2} F(\hbar, s, t)}
$$

and therefore $\tau_{0}$ and $\tau_{1}$ behave as

$$
\tau_{0}(\hbar ; s ; t)=e^{\hbar^{-2} F(\hbar, N s, t)}, \quad \tau_{1}(\hbar ; s ; t)=e^{\hbar^{-2} F(\hbar, N s+\hbar, t)} .
$$

Substituting this into (2.39) and comparing the result with (3.41) and (3.42), we have

$$
\frac{\partial F(\hbar, N s, t)}{\partial t_{n}}=v_{n}(s, t)+O(\hbar), \quad-\frac{\hbar}{N} \frac{\partial F(\hbar, N s, t)}{\partial s}=\hbar \phi_{0}(s, t)+O\left(\hbar^{2}\right) .
$$

Hence $\log \tau_{\operatorname{dcmKP}}(s ; t)=\left.F(\hbar, N s, t)\right|_{\hbar=0}$, which satisfies (3.19) and (3.46).

\section{A Form of $\boldsymbol{P}(s), \mathcal{P}(s)$ and proof of Proposition 2 (ii)}

In the main text we assumed that operator $P(s)$ of the cmKP hierarchy does not have the 0-th order term as in (2.2). We also put similar requirement (3.2) to $\mathcal{P}(s)$ of the dcmKP hierarchy. At first glance, these assumptions might seem artificial but in fact they are not restriction as we show in this appendix.

Assume that $L(s)$ is of the form $(2.1)$ and that $P(s)$ has the form

$$
\begin{aligned}
P(s ; x, t) & :=p_{0}(s, x, t) \partial^{m_{s}}+\cdots+p_{m_{s}-1}(s, t) \partial+p_{m_{s}}(s, x, t) \\
& =\sum_{n=0}^{m_{s}} p_{n}(s, x, t) \partial^{m_{s}-n}, \quad p_{0}(s, x, t) \neq 0,
\end{aligned}
$$

instead of the form (2.2). Assume further that $(L(s), P(s))_{s \in S}$ satisfies the system (2.3), (2.4) and (2.5). We show that there exists a function $f(s)=f(s, x, t)$ which satisfies

- The pair $\left(\tilde{L}(s):=f(s)^{-1} L(s) f(s), \tilde{P}(s):=f(s+1)^{-1} P(s) f(s)\right)_{s \in S}$ is a solution of the cmKP hierarchy.

- $\tilde{P}(s)$ does not have the 0 -th order term:

$$
\tilde{P}(s)=\tilde{p}_{0}(s) \partial^{m_{s}}+\cdots+\tilde{p}_{m_{s}-1}(s) \partial+\tilde{p}_{m_{s}}(s), \quad \tilde{p}_{m_{s}}(s)=0 .
$$

In this sense we can assume without loss of generality that $p_{m_{s}}(s)=0$ in $(2.2)$.

The following is the basic lemma:

Lemma 1. Let $Q=\sum_{j=0}^{N} q_{j}(x) \partial^{N-j}$ be a differential operator and $f(x)$ is a function. Then the 0 -th order term of the composition $Q \circ f$ is the function $Q(f)$ obtained by applying $Q$ on $f$.

This is a direct consequence of the Leibniz rule: $\partial^{k} \circ f=\sum_{r=0}^{k}\left(\begin{array}{l}k \\ r\end{array}\right) f^{(k-r)} \partial^{r}$.

Hence the second condition (A.2) for the function $f(s, t)$ is equivalent to

$$
P(s)(f(s))=0 .
$$

Let us introduce an operator $C_{n}(s)$ by

$$
f(s)^{-1}\left(\partial_{t_{n}}-B_{n}(s)\right) f(s)=\partial_{t_{n}}-C_{n}(s),
$$




$$
\text { i.e., } \quad C_{n}(s)=f(s)^{-1} B_{n}(s) f(s)-f(s)^{-1} \frac{\partial f(s)}{\partial t_{n}} \text {. }
$$

Then, it follows from $(2.3),(2.4)$ and $(2.5)$ that the pair $(\tilde{L}(s), \tilde{P}(s))_{s \in S}$ satisfies the following equations:

$$
\begin{aligned}
& {\left[\tilde{L}(s), \partial_{t_{n}}-C_{n}(s)\right]=0,} \\
& \tilde{L}(s+1) \tilde{P}(s)=\tilde{P}(s) \tilde{L}(s), \\
& \left(\partial_{t_{n}}-C_{n}(s+1)\right) \tilde{P}(s)=\tilde{P}(s)\left(\partial_{t_{n}}-C_{n}(s)\right) .
\end{aligned}
$$

Hence if $C_{n}(s)=\left(\tilde{L}(s)^{n}\right)_{>0}$, then $(\tilde{L}(s), \tilde{P}(s))$ is a solution of the cmKP hierarchy. By Lemma 1 we have

$$
C_{n}(s)=\left(f(s)^{-1} B_{n}(s) f(s)\right)_{>0}+f(s)^{-1} B_{n}(s)(f(s))-f(s)^{-1} \frac{\partial f(s)}{\partial t_{n}} .
$$

Therefore the condition $C_{n}(s)=\left(\tilde{L}(s)^{n}\right)_{>0}$ is equivalent to

$$
\left(\partial_{t_{n}}-B_{n}(s)\right)(f(s))=0 .
$$

So, we have to find a function $f(s)$ satisfying (A.3) and (A.8). This is done inductively as follows. First solve equation (A.3) for $t_{1}=t_{2}=\cdots=0$ but for arbitrary $x$. We denote the solution by $f_{0}(s, x)$ :

$$
P\left(s ; x, t_{1}=t_{2}=\cdots=0\right) f_{0}(s, x)=0, \quad f_{0}(s, 0)=1 .
$$

Function $f_{1}\left(s, x, t_{1}\right)=f_{0}\left(s, x+t_{1}\right)$ satisfies (A.3) as well as (A.8) with $n=1$ for $t_{2}=t_{3}=\cdots=0$.

Suppose we have function $f_{m}\left(s, x, t_{1}, \ldots, t_{m}\right)$ which satisfies (A.3) and (A.8) with $n=$ $1, \ldots, m$ and $t_{k}=0(k>m)$. We can solve the Cauchy problem

$$
\begin{aligned}
& \frac{\partial}{\partial t_{m+1}} f_{m+1}\left(s, x, t_{1}, \ldots, t_{m+1}\right) \\
& \quad-B_{m+1}\left(s, x, t_{1}, \ldots, t_{m+1}, 0,0, \ldots\right) f_{m+1}\left(s, x, t_{1}, \ldots, t_{m+1}\right)=0, \\
& \quad f_{m+1}\left(s, x, t_{1}, \ldots, t_{m}, 0\right)=f_{m}\left(s, x, t_{1}, \ldots, t_{m}\right),
\end{aligned}
$$

with respect to $t_{m+1}$. By (2.7) and (2.5) for $n=m+1$, the solution $f_{m+1}$ of (A.10) satisfies (A.3) and (A.8) for $n=1, \ldots, m+1$ and $t_{k}=0(k>m+1)$.

The desired function $f(s, t)=f\left(s, x, t_{1}, t_{2}, \ldots\right)$ is defined by the inductive limit of the sequence $f_{n}\left(s, x, t_{1}, t_{2}, \ldots, t_{n}\right)$.

The second statement of Proposition 2 is proved in the same way. Suppose that a solution $\left(L^{\mathrm{mKP}}(s), P^{\mathrm{mKP}}(s)\right)_{s \in S}$ of the mKP hierarchy and a sequence $\left\{\left(f^{(0)}(s), \ldots, f^{\left(m_{s}-1\right)}(s)\right)\right\}_{s \in S}$ of non-zero constant vectors are given. Replace $L(s), P(s)$ and $B_{n}(s)$ in the above argument by $L^{\mathrm{mKP}}(s), P^{\mathrm{mKP}}(s)$ and $B_{n}^{\mathrm{mKP}}(s)$ respectively. (See $(2.13),(2.15)$ and (2.14).) If we solve equation (A.9) under the initial condition

$$
\partial^{k} f_{0}(s, 0)=f^{(k)}(s), \quad k=0, \ldots, m_{s}-1, \quad\left(s \in S^{\prime}\right),
$$

we obtain a function $f(s)=f(s, x, t)$ such that

$$
\partial^{k} f_{0}(s ; 0,0)=f^{(k)}(s) \quad \text { for all } \quad s \in S, \quad 0 \leqq k<m_{s},
$$

and $\left(L(s):=f(s)^{-1} L^{\mathrm{mKP}}(s) f(s), P(s):=f(s+1)^{-1} P^{\mathrm{mKP}}(s) f(s)\right)_{s \in S}$ is a solution of the cmKP hierarchy.

We proceed to the case of the dcmKP hierarchy. 
Lemma 2. Let $(\mathcal{L}(s), \mathcal{P}(s))$, where

$$
\begin{aligned}
& \mathcal{L}(s)=\mathcal{L}(k ; s, t)=k+\sum_{n=0}^{\infty} u_{n+1}(s, t) k^{-n}, \\
& \mathcal{P}(s)=\mathcal{P}(k ; s, t)=p_{0}(s, t) k^{N}+\cdots+p_{N}(s, t),
\end{aligned}
$$

be a solution of the dcmKP hierarchy. If $\varphi(s, t)$ is a function that satisfies the system of equations $^{2}$

$$
\frac{\partial \varphi(s, t)}{\partial t_{n}}=-\mathcal{B}_{n}\left(-\frac{\partial \varphi(s, t)}{\partial x} ; s, t\right), \quad n \geq 1,
$$

then the pair $(\tilde{\mathcal{L}}(s), \tilde{\mathcal{P}}(s))$, where

$$
\begin{aligned}
& \tilde{\mathcal{L}}(k ; s, t)=e^{\mathrm{ad} \varphi(s, t)} \mathcal{L}(k ; s, t)=\mathcal{L}\left(k-\frac{\partial \varphi(s, t)}{\partial x} ; s, t\right) \\
& \tilde{\mathcal{P}}(k ; s, t)=e^{\partial \varphi(s, t) / \partial s} e^{\operatorname{ad} \varphi(s, t)} \mathcal{L}(k ; s, t)=e^{\partial \varphi(s, t) / \partial s} \mathcal{P}\left(k-\frac{\partial \varphi(s, t)}{\partial x} ; s, t\right),
\end{aligned}
$$

is also a solution of the dcmKP hierarchy.

Proof. First, observe that

$$
\tilde{\mathcal{B}}_{n}=\left(\tilde{\mathcal{L}}^{n}\right)_{>0}=e^{\operatorname{ad} \varphi} \mathcal{B}_{n}-\mathcal{B}_{n}\left(-\frac{\partial \varphi}{\partial x}\right)
$$

Therefore, we have

$$
\begin{aligned}
\frac{\partial \tilde{\mathcal{L}}}{\partial t_{n}} & =\left\{\frac{\partial \varphi}{\partial t_{n}}, \tilde{\mathcal{L}}\right\}+e^{\operatorname{ad} \varphi}\left\{\mathcal{B}_{n}, \mathcal{L}\right\} \\
& =\left\{-\mathcal{B}_{n}\left(-\frac{\partial \varphi}{\partial x}\right)+e^{\operatorname{ad} \varphi} \mathcal{B}_{n}, \tilde{\mathcal{L}}\right\}=\left\{\tilde{\mathcal{B}}_{n}, \tilde{\mathcal{L}}\right\}
\end{aligned}
$$

Similarly,

$$
\frac{\partial \tilde{\mathcal{L}}}{\partial s}=\left\{\frac{\partial \varphi}{\partial s}+e^{\operatorname{ad} \varphi} \log \mathcal{P}, \tilde{\mathcal{L}}\right\}=\{\log \tilde{\mathcal{P}}, \tilde{\mathcal{L}}\}
$$

Finally,

$$
\begin{aligned}
\frac{\partial \log \tilde{\mathcal{P}}}{\partial t_{n}}= & \frac{\partial^{2} \varphi}{\partial t_{n} \partial s}+\left\{\frac{\partial \varphi}{\partial t_{n}}, e^{\operatorname{ad} \varphi} \log \mathcal{P}\right\}+e^{\operatorname{ad} \varphi}\left(\frac{\partial \mathcal{B}_{n}}{\partial s}-\left\{\log \mathcal{P}, \mathcal{B}_{n}\right\}\right) \\
= & \left\{\frac{\partial \varphi}{\partial t_{n}}, \log \tilde{\mathcal{P}}\right\}+\frac{\partial^{2} \varphi}{\partial s \partial t_{n}}+\frac{\partial}{\partial s}\left(e^{\operatorname{ad} \varphi} \mathcal{B}_{n}\right)-\left\{\frac{\partial \varphi}{\partial s}, e^{\operatorname{ad} \varphi} \mathcal{B}_{n}\right\} \\
& -\left\{e^{\operatorname{ad} \varphi} \log \mathcal{P}, e^{\operatorname{ad} \varphi} \mathcal{B}_{n}\right\} \\
= & \frac{\partial \tilde{\mathcal{B}}_{n}}{\partial s}-\left\{\log \tilde{\mathcal{P}}, \tilde{\mathcal{B}}_{n}\right\} .
\end{aligned}
$$

\footnotetext{
${ }^{2}$ The equation when $n=1$ is a tautology.
} 
Lemma 3. Let $(\mathcal{L}(s), \mathcal{P}(s))$ be as in the lemma above. If $\mathcal{P}(k ; s, t)$ has a root $\psi(s, t)$ as a polynomial of $k$, the system

$$
\frac{\partial \varphi(s, t)}{\partial t_{n}}=-\mathcal{B}_{n}(\psi(s, t) ; s, t), \quad n \geq 1,
$$

has a solution, unique up to a function of $s$.

In particular, (A.13) for $n=1$ implies that $\psi(s, t)=-\frac{\partial \varphi}{\partial x}$. Hence the function satisfying (A.12) is obtained and we can gauge away the constant term of $\mathcal{P}(s)$ according to Lemma 2.

Proof. Let us factorize $\mathcal{P}(k ; s, t)$ as $\mathcal{P}(k ; s, t)=p_{0}(s, t) \prod_{i=1}^{N}\left(k-\psi_{i}(s, t)\right)$. Differentiating by $t_{n}$, we have

$$
\frac{\partial \log \mathcal{P}}{\partial t_{n}}=\sum_{i=1}^{N} \frac{-\frac{\partial \psi_{i}}{\partial t_{n}}}{k-\psi_{i}}+\frac{\partial}{\partial t_{n}} \log p_{0} .
$$

The left hand side is, due to (3.5),

$$
\begin{aligned}
\frac{\partial \log \mathcal{P}}{\partial t_{n}} & =\frac{\partial \mathcal{B}_{n}}{\partial s}-\frac{\partial \log \mathcal{P}}{\partial k} \frac{\partial \mathcal{B}_{n}}{\partial x}+\frac{\partial \log \mathcal{P}}{\partial x} \frac{\partial \mathcal{B}_{n}}{\partial k} \\
& =\frac{\partial \mathcal{B}_{n}}{\partial s}-\frac{\partial \mathcal{B}_{n}}{\partial x} \sum_{i=1}^{N} \frac{1}{k-\psi_{i}}+\frac{\partial \mathcal{B}_{n}}{\partial k} \sum_{i=1}^{N} \frac{-\frac{\partial \psi_{i}}{\partial x}}{k-\psi_{i}}
\end{aligned}
$$

Substituting this into (A.14), multiplying $\frac{\partial \mathcal{B}_{m}}{\partial k}$, subtracting the same equation with $m$ and $n$ interchanged, we have

$$
\left\{\mathcal{B}_{n}, \mathcal{B}_{m}\right\}\left(\psi_{i} ; s, t\right)=\frac{\partial \mathcal{B}_{n}}{\partial k}\left(\psi_{i} ; s, t\right) \frac{\partial \psi_{i}(s, t)}{\partial t_{m}}-\frac{\partial \mathcal{B}_{m}}{\partial k}\left(\psi_{i} ; s, t\right) \frac{\partial \psi_{i}(s, t)}{\partial t_{n}}
$$

by comparing the residue at $k=\psi_{i}$. Using this equation, we can check the consistency of the system (A.13) as follows:

$$
\begin{aligned}
\frac{\partial}{\partial t_{m}} \mathcal{B}_{n}(\psi(s, t) ; s, t)-\frac{\partial}{\partial t_{n}} \mathcal{B}_{m}(\psi(s, t) ; s, t) \\
=\left(\frac{\partial \mathcal{B}_{n}}{\partial t_{m}}-\frac{\partial \mathcal{B}_{m}}{\partial t_{n}}\right)(\psi(s, t) ; s, t) \\
\quad+\frac{\partial \mathcal{B}_{n}}{\partial k}(\psi(s, t) ; s, t) \frac{\partial \psi(s, t)}{\partial t_{m}}-\frac{\partial \mathcal{B}_{m}}{\partial k}(\psi(s, t) ; s, t) \frac{\partial \psi(s, t)}{\partial t_{n}} \\
=\left(\frac{\partial \mathcal{B}_{n}}{\partial t_{m}}-\frac{\partial \mathcal{B}_{m}}{\partial t_{n}}+\left\{\mathcal{B}_{n}, \mathcal{B}_{m}\right\}\right)(\psi(s, t) ; s, t)=0 .
\end{aligned}
$$

Therefore, the system (A.13) has a solution $\varphi(s, t)$ unique up to a function of $s$.

\section{B Difference operator formalism}

When the set $\left\{n_{s}\right\}_{s \in S}$ is equal to the whole set of integer numbers $\mathbb{Z}$, we can formulate the cmKP hierarchy in terms of difference operators. In fact in this case the cmKP hierarchy can be thought of as the "half" of the Toda lattice hierarchy of Ueno and Takasaki [23] whose dependence on half of the time variables are suppressed.

In this appendix, we first present the difference operator formalism of the cmKP hierarchy and then show in Appendix D that it is equivalent to the cmKP hierarchy in the main text. 
We also introduce a gauge parameter $\alpha, \alpha \neq 0$. (See [14] for the gauge parameter of the Toda lattice hierarchy.)

Let $\boldsymbol{L}$ be a difference operator of the form

$$
\boldsymbol{L}=b_{0}(s, t) e^{\partial_{s}}+b_{1}(s, t)+b_{2}(s, t) e^{-\partial_{s}}+\cdots=\sum_{j=0}^{\infty} b_{j}(s, t) e^{(1-j) \partial_{s}},
$$

where $e^{k \partial_{s}}$ is the $k$-step shift operator $e^{k \partial_{s}} f(s)=f(k+s)$ and $t=\left(t_{1}, t_{2}, t_{3}, \ldots\right)$ is a sequence of continuous variables. We assume that $b_{0}$ never vanishes: $b_{0}(s, t) \neq 0$.

We call the following system the difference operator formalism of the cmKP hierarchy with gauge parameter $\alpha$ :

$$
\frac{\partial \boldsymbol{L}}{\partial t_{n}}=\left[\boldsymbol{B}_{n}, \boldsymbol{L}\right]
$$

Here $\boldsymbol{B}_{n}$ is a difference operator defined by

$$
\boldsymbol{B}_{n}:=\left(\boldsymbol{L}^{n}\right)_{\geq 0}-\alpha\left(\boldsymbol{L}^{n}\right)_{0},
$$

where $(\cdot)_{\geq 0}$ and $(\cdot)_{0}$ are projections of difference operators: for $A=\sum_{j} a_{j}(s) e^{j \partial_{s}}$,

$$
A_{\geq 0}:=\sum_{j \geq 0} a_{j}(s) e^{j \partial_{s}}, \quad A_{0}:=a_{0}(s), \quad A_{<0}:=\sum_{j<0} a_{j}(s) e^{j \partial_{s}} .
$$

By the same argument as in $[23, \S 1]$, the Lax representation (B.2) of the cmKP hierarchy is equivalent to the Zakharov-Shabat (or zero-curvature) representations:

$$
\begin{aligned}
& {\left[\partial_{t_{m}}-\boldsymbol{B}_{m}, \partial_{t_{n}}-\boldsymbol{B}_{n}\right]=0,} \\
& {\left[\partial_{t_{m}}-\boldsymbol{B}_{m}^{c}, \partial_{t_{n}}-\boldsymbol{B}_{n}^{c}\right]=0,}
\end{aligned}
$$

where

$$
\boldsymbol{B}_{n}^{c}=\boldsymbol{B}_{n}-\boldsymbol{L}^{n}=-\left(\boldsymbol{L}^{n}\right)_{<0}-\alpha\left(\boldsymbol{L}^{n}\right)_{0} .
$$

Example 1. Dispersionless limit of the case $\alpha=1 / 2$ is (3.34). It is related to the Löwner equation. See [22].

The proof of the following proposition is the same as those of Theorem 1.2 of [23].

Proposition 13. For each solution of the cmKP hierarchy with gauge parameter $\alpha$, there exists a difference operator $\hat{\boldsymbol{W}}$ of the following form with coefficients $w_{0}(s, t)=e^{-\alpha \varphi(s, t)}$ and $w_{j}(s, t)$ :

$$
\hat{\boldsymbol{W}}=e^{-\alpha \varphi(s, t)}+w_{1}(s, t) e^{-\partial_{s}}+\cdots=\sum_{j=0}^{\infty} w_{j}(s, t) e^{-j \partial_{s}},
$$

satisfying the equations

$$
\boldsymbol{L}=\hat{\boldsymbol{W}} e^{\partial_{s}} \hat{\boldsymbol{W}}^{-1}, \quad \frac{\partial \hat{\boldsymbol{W}}}{\partial t_{n}}=\boldsymbol{B}_{n}^{c} \hat{\boldsymbol{W}}
$$

where the operators $\boldsymbol{B}_{n}^{c}$ are defined by (B.7).

We call $\hat{\boldsymbol{W}}$ the wave matrix. 
Proposition 14. (i) The operator $\hat{\boldsymbol{W}}$ in Proposition 13 and the function $\varphi(s, t)$ satisfy the following bilinear equation for arbitrary $t$ and $t^{\prime}$ :

$$
\boldsymbol{W}(t) \boldsymbol{W}\left(t^{\prime}\right)^{-1}=e^{(1-\alpha)\left(\varphi(s, t)-\varphi\left(s, t^{\prime}\right)\right)}+(\text { strictly upper triangular }),
$$

where $\boldsymbol{W}(t)=\hat{\boldsymbol{W}}(t) \exp \left(\sum_{n=1}^{\infty} t_{n} e^{n \partial_{s}}\right)$ and the "(strictly upper triangular)" part is an operator of the form $\sum_{n>0} a_{n}(s) e^{n \partial_{s}}$.

(ii) Conversely, if a function $\varphi(s, t)$ and an operator $\hat{\boldsymbol{W}}$ of the form (B.8) satisfies the equation (B.10), then the operator $\boldsymbol{L}$ defined by $\boldsymbol{L}=\hat{\boldsymbol{W}} e^{\partial_{s}} \hat{\boldsymbol{W}}^{-1}$ is a solution of the cmKP hierarchy (B.2).

The proof is essentially the same as the proof of Proposition 1.4 and Theorem 1.5 of [23].

By means of $\varphi(s, t)$ in Proposition 13, we can change the gauge as follows.

Proposition 15. Let $\boldsymbol{L}$ be a solution of the cmKP hierarchy (B.2) with gauge parameter $\alpha$ and $\varphi$ be the function defined in Proposition 13. Then the difference operator defined by

$$
\tilde{\boldsymbol{L}}:=e^{(\alpha-\beta) \varphi(s, t)} \boldsymbol{L} e^{-(\alpha-\beta) \varphi(s, t)}
$$

satisfies equation (B.2) with gauge parameter $\beta$. Here $\beta$ can be 0 .

Simple calculation is sufficient to check (B.2) for $\tilde{\boldsymbol{L}}$. We have only to note that the truncating operations $(\cdot)_{\geq 0},(\cdot)_{0}$ (cf. (B.4)) commute with the adjoint operation $e^{(\alpha-\beta) \varphi(s, t)}(\cdot) e^{-(\alpha-\beta) \varphi(s, t)}$.

\section{The cmKP hierarchy with a gauge parameter}

As is naturally expected from Section $\mathrm{B}$, we can introduce a gauge parameter $\alpha(\alpha \neq 0)$ in the cmKP hierarchy when $\left\{n_{s}\right\}_{s \in S}=\mathbb{Z}$. In this case operator $L(s)$ has the form as in (2.1) but $P(s)$ has a 0 -th order term:

$$
\begin{aligned}
& L(s)=L(t ; s):=\partial+u_{1}(s, t)+u_{2}(s, t) \partial^{-1}+u_{3}(s, t) \partial^{-2}+\cdots, \\
& P(s)=P(s, t):=p_{0}(s, t) \partial+p_{1}(s, t),
\end{aligned}
$$

which satisfy the condition

$$
(1-\alpha) p_{0}(s, t) u_{1}(s, t)+\alpha p_{1}(s, t)=0 .
$$

This condition is equivalent to saying that $B_{1}(s)$ defined later is equal to $\partial$. The cmKP hierarchy in Section 2.1 is recovered when $\alpha=1$.

We introduce operators $P^{(n)}(s)$ which play the role of the $n$-step shift operators:

$$
P^{(n)}(s):= \begin{cases}P(s+n-1) \cdots P(s+1) P(s), & n>0, \\ 1, & n=0, \\ P(s+n)^{-1} \cdots P(s-2)^{-1} P(s-1)^{-1}, & n<0 .\end{cases}
$$

The fundamental properties of $P^{(n)}(s)$ are the following:

Lemma 4. (i) Any microdifferential operator $Q$ has a unique expansion of the form

$$
Q=\sum_{\nu \in \mathbb{Z}} a_{\nu} P^{(\nu)}(s)
$$

If $Q$ is a $n$-th order differential operator, the sum is taken over $0 \leqq \nu \leqq n$.

(ii)

$$
P^{(m)}(s+n) P^{(n)}(s)=P^{(m+n)}(s) .
$$


According to Lemma 4 (i), the operator $L(s)^{n}$ is expanded as:

$$
L(s)^{n}=\sum_{j=0}^{\infty} b_{j}^{(n)}(s, t) P^{(n-j)}(s) .
$$

For example, since $L(s)=p_{0}(s, t)^{-1} P(s)-p_{0}(s, t)^{-1} p_{1}(s, t)+u_{1}(s, t)+\cdots$, we have

$$
b_{0}^{(1)}(s, t)=p_{0}(s, t)^{-1}, \quad b_{1}^{(1)}(s, t)=-p_{0}(s, t)^{-1} p_{1}(s, t)+u_{1}(s, t) .
$$

We define operator $B_{n}$ by

$$
\begin{aligned}
B_{n}(s) & :=\left(L(s)^{n}\right)_{\geq 0}-\alpha b_{n}^{(n)}(s, t) \\
& =\sum_{j=0}^{n-1} b_{j}^{(n)}(s, t) P^{(n-j)}(s)+(1-\alpha) b_{n}^{(n)}(s, t) .
\end{aligned}
$$

Here $(\cdot)_{\geq 0}$ is the projection of a microdifferential operator to the differential operator part. It is easy to see that condition (C.3) is equivalent to $B_{1}(s)=\partial$ and that $B_{n}(s)=\left(L(s)^{n}\right)_{>0}$ when $\alpha=1$.

The definition of the cmKP hierarchy with a gauge parameter $\alpha$ is the same as the usual one, i.e., (2.3), (2.4) and (2.5).

Proposition 16. The pair $(\tilde{L}(s), \tilde{P}(s))_{s \in \mathbb{Z}}$ of sequences of differential operators

$$
\begin{aligned}
\tilde{L}(s) & :=e^{(\alpha-\beta) \varphi(s, t)} L(s) e^{-(\alpha-\beta) \varphi(s, t)} \\
\tilde{P}(s) & :=e^{(\alpha-\beta) \varphi(s+1, t)} P(s) e^{-(\alpha-\beta) \varphi(s, t)}
\end{aligned}
$$

for $s \in \mathbb{Z}$ is a solution of the system (2.3), (2.4), (2.5) with gauge parameter $\beta$. Here $\beta$ can be 0 .

Proof. Let us check the condition (C.3) first. The operators $\tilde{L}(s)$ and $\tilde{P}(s)$ have the form

$$
\begin{aligned}
& \tilde{L}(s)=\partial+\tilde{u}_{1}(s)+\tilde{u}_{2}(s) \partial^{-1}+\cdots, \\
& \tilde{P}(s)=\tilde{p}_{0}(s) \partial+\tilde{p}_{1}(s),
\end{aligned}
$$

where

$$
\begin{aligned}
& \tilde{u}_{1}(s)=u_{1}(s)-(\alpha-\beta) \varphi^{\prime}(s, t) \\
& \tilde{p}_{0}(s)=e^{(\alpha-\beta)(\varphi(s+1, t)-\varphi(s, t))} p_{0}(s), \\
& \tilde{p}_{1}(s)=e^{(\alpha-\beta)(\varphi(s+1, t)-\varphi(s, t))}\left(p_{1}(s)-(\alpha-\beta) p_{0}(s) \varphi^{\prime}(s, t)\right) .
\end{aligned}
$$

Here $\varphi^{\prime}(s, t)=\partial \varphi(s, t) / \partial x=\partial \varphi(s, t) / \partial t_{1}=b_{1}^{(1)}(s, t)$. Using the explicit form (C.8), we can check that $(1-\beta) \tilde{p}_{0}(s, t) \tilde{u}_{1}(s, t)+\beta \tilde{p}_{1}(s, t)=0$.

The operator $P^{(n)}(s)$ defined by (C.4) transforms as

$$
P^{(n)}(s) \mapsto \tilde{P}^{(n)}(s):=e^{(\alpha-\beta) \varphi(s+n, t)} P^{(n)}(s) e^{-(\alpha-\beta) \varphi(s, t)}
$$

by the transformation (C.10). Hence $(\tilde{L}(s))^{n}$ is expanded as

$$
\begin{aligned}
& (\tilde{L}(s))^{n}=\sum_{j=0}^{\infty} \tilde{b}_{j}^{(n)}(s) \tilde{P}^{(n-j)}(s), \\
& \tilde{b}_{j}^{(n)}(s):=e^{(\alpha-\beta)(\varphi(s, t)-\varphi(s+n-j, t))} b_{j}^{(n)}(s) .
\end{aligned}
$$


Particularly, $\tilde{b}_{n}^{(n)}(s)=b_{n}^{(n)}(s)$, which implies

$$
\begin{aligned}
\tilde{B}_{n}(s) & :=(\tilde{L}(s))_{\geq 0}^{n}-\beta(\tilde{L}(s))_{0}^{n} \\
& =e^{(\alpha-\beta) \varphi(s, t)} B_{n}(s) e^{-(\alpha-\beta) \varphi(s, t)}+(\alpha-\beta) b_{n}^{(n)}(s) .
\end{aligned}
$$

It remains to check (2.3) and (2.5) by straightforward computation. (Equation (2.4) is obvious from the definition of $\tilde{L}(s)$ and $\tilde{P}(s),($ C.10).)

Corollary 4. The cmKP hierarchies with different gauge parameters are equivalent through the transformation (C.10)

If $\beta=0$ in Proposition 16, the resulting system is the mKP hierarchy. Proposition 2 is the case when $(\alpha, \beta)=(1,0)$. See Section 2.2.

\section{Equivalence of two formulations}

The two formalisms of the cmKP hierarchy discussed in Appendix B and Appendix $\mathrm{C}$ are equivalent. The proof is almost straightforward computation but lengthy.

Rewriting the difference operator formalism to the differential operator formalism is essentially the same as the procedure described in $\S 1.2$ of [23], where the KP hierarchy is embedded in the Toda lattice hierarchy. Assume that a solution $\boldsymbol{L}=\sum_{j=0}^{\infty} b_{j}(s, t) e^{(1-j) \partial_{s}}$ of the system (B.2) is given. The idea is to interpret the operator $\partial_{t_{1}}-\boldsymbol{B}_{1}$ as the operator $b_{0}(s, t)\left(P(s)-e^{\partial_{s}}\right)$. Namely, we define the operator $P$ by

$$
P(s):=b_{0}(s, t)^{-1}\left(\partial-(1-\alpha) b_{1}(s, t)\right),
$$

where $t_{1}$ is replaced by $t_{1}+x$. (We do not write the dependence on $x$ explicitly.) Using the operator $P^{(n)}(s)$ defined by (C.4), we define the $L$ operator by

$$
\begin{aligned}
L(s) & :=\sum_{j=0}^{\infty} b_{j}(s, t) P^{(1-j)}(s) \\
& =b_{0}(s, t) P(s)+b_{1}(s, t)+b_{2}(s, t) P^{(-1)}(s)+\cdots \\
& =\partial+\alpha b_{1}(s, t)+\cdots
\end{aligned}
$$

The condition (C.3) is automatically satisfied, thus we have $B_{1}(s)=\partial$.

First we prove (2.4). The left hand side of (2.4) is

$$
L(s+1) P(s)=\sum_{j=0}^{\infty} b_{j}(s+1) P^{(2-j)}(s),
$$

by the definition (C.4). The right hand side of (2.4) is

$$
\begin{aligned}
P(s) L(s) & =P(s) \sum_{j=0}^{\infty} b_{j}(s) P^{(1-j)}(s) \\
& =b_{0}(s)^{-1} \sum_{j=0}^{\infty}\left(\frac{\partial b_{j}(s)}{\partial x}+b_{j}(s) \partial-(1-\alpha) b_{j}(s) b_{1}(s)\right) P^{(1-j)}(s) .
\end{aligned}
$$


Since $\partial b_{j}(s) / \partial x=\partial b_{j}(s) / \partial t_{1}$, the Lax equation (B.2) with $n=1$ gives information on $\partial b_{j}(s) / \partial x$. Comparing the coefficients of $e^{(1-j) \partial_{s}}$ in (B.2), we have

$$
\begin{aligned}
\frac{\partial b_{j}(s)}{\partial x}= & b_{0}(s) b_{j+1}(s+1)-b_{0}(s-j) b_{j+1}(s) \\
& +(1-\alpha) b_{1}(s) b_{j}(s)-(1-\alpha) b_{1}(s+1-j) b_{j}(s) .
\end{aligned}
$$

Substituting it into (D.4) and using the property (C.6) of $P^{(j)}(s)$, we have

$$
P(s) L(s)=\sum_{j=0}^{\infty} b_{j}(s+1) P^{(2-j)}(s)
$$

which, together with (D.3), proves (2.4). The following formula is a consequence of (2.4):

$$
L(s+m) P^{(m)}(s)=P^{(m)}(s) L(s) .
$$

The proof of (2.5) is almost the same. Note that if we expand $\boldsymbol{L}^{n}$ as

$$
\boldsymbol{L}^{n}=\sum_{j=0}^{\infty} b_{j}^{(n)}(s) e^{(n-j) \partial_{s}}
$$

operator $L(s)^{n}$ is expanded as (C.7) with the same coefficients $b_{j}^{(n)}(s)$ as in (D.8). This is proved by induction with the help of (2.4) proved above and (C.6).

Hence the coefficients $b_{j}^{(n)}(s)$ in (C.9) are the same as in (D.8). Using this fact and (B.5) $m=1$, we can prove (2.6), i.e., (2.5). We omit details which are similar to the above proof of (2.4). The formula

$$
\begin{aligned}
& \left(\partial_{t_{n}}-B_{n}(s+m)\right) P^{(m)}(s)=P^{(m)}(s)\left(\partial_{t_{n}}-B_{n}(s)\right), \\
& \text { i.e., } \quad \frac{\partial P^{(m)}(s)}{\partial t_{n}}=B_{n}(s+m) P^{(m)}(s)-P^{(m)}(s) B_{n}(s),
\end{aligned}
$$

derived from (2.5) shall be used in the following step.

Let us proceed to the proof of the Lax equation (2.3). Its left hand side is

$$
\begin{aligned}
\frac{\partial L(s)}{\partial t_{n}}= & \sum_{j=0}^{\infty} \frac{\partial b_{j}(s)}{\partial t_{n}} P^{(1-j)}(s) \\
& +\sum_{j=0}^{\infty} \sum_{k=0}^{n} b_{j}(s) b_{k}^{(n)}(s+1-j) P^{(n+1-j-k)}(s)-L(s) B_{n}(s) .
\end{aligned}
$$

(We used (D.10) and (C.6).) On the other hand, (D.7) and (C.6) imply

$$
\left[B_{n}(s), L(s)\right]=\sum_{j=0}^{n} \sum_{k=0}^{\infty} b_{j}^{(n)}(s) b_{k}(s+n-j) P^{(n+1-j-k)}(s)-L(s) B_{n}(s) .
$$

In order to prove that (D.11) and (D.12) are equal, we have only to show

$$
\frac{\partial b_{l}(s)}{\partial t_{n}}=\sum_{\substack{0 \leq j \leq n, 0 \leq k \\ j+k=l+n}}\left(b_{j}^{(n)}(s) b_{k}(s+n-j)-b_{k}(s) b_{j}^{(n)}(s+1-k)\right),
$$


which is nothing but the coefficient of $e^{(1-l) \partial_{s}}$ of (B.2). Thus we have proved that a solution of the difference operator equations (B.2) gives a solution of the system (2.3), (2.4) and (2.5).

Conversely, when a solution $(L(s), P(s))_{s \in \mathbb{Z}}$ of the system $(2.3),(2.4),(2.5)$ is given and $L(s)$ is expanded as

$$
L(s)=\sum_{j=0}^{\infty} b_{j}(s) P^{(1-j)}(s),
$$

then the $\boldsymbol{L}$ operator defined by (B.1) satisfies the system (B.2). Note that, due to condition (C.3), $B_{1}(s)=\partial$, which means that $t_{1}$ and $x$ always appear in the form $t_{1}+x$. Hence we can eliminate $x$ by just replacing $t_{1}+x$ by $t_{1}$. The Lax equation (B.2) for the difference operator is proved by tracing back the above proof of (2.3).

Remark 5. This correspondence holds also for the case $\alpha=0$. If $p_{0}(s)$ is normalized to 1 , the system $(2.3),(2.4),(2.5)$ is the mKP hierarchy in $[2,17]$. Its equivalence to the system $(\mathrm{B} .2)^{3}$ was proved in [2] by a different method.

\section{Acknowledgments}

The work of T. Takebe is supported in part by JSPS grant 18540210. He is also grateful to Saburo Kakei and Kanehisa Takasaki for their interest and comments. The work of L.P. Teo is supported in part by MMU funding PR/2006/0590. She would also like to thank Academia Sinica of Taiwan for its hospitality during her stay, when part of this work was done.

[1] Chang J.H., Tu M.H., On the Miura map between the dispersionless KP and dispersionless modified KP hierarchies, J. Math. Phys., 2000, V.41, 5391-5406, solv-int/9912016.

[2] Dickey L.A., Modified KP and discrete KP, Lett. Math. Phys., 1999, V.48, 277-289, solv-int/9902008.

[3] Date E., Kashiwara M., Jimbo M., Miwa T., Transformation groups for soliton equations, in Nonlinear Integrable Systems - Classical Theory and Quantum Theory, Singapore, World Scientific, 1983, 39-119.

[4] Duren P.L., Univalent functions, Grundlehren der Mathematischen Wissenschaften, Vol. 259, New York, Springer-Verlag, 1983.

[5] Kashiwara M., Miwa T., Transformation groups for soliton equations. I. The $\tau$ function of the KadomtsevPetviashvili equation, Proc. Japan Acad. Ser. A Math. Sci., 1981, V.57, 342-347.

[6] Kac V.G., Peterson D.H., Lectures on the infinite wedge-representation and the MKP hierarchy, in Systèmes dynamiques non linéaires: intégrabilité et comportement qualitatif, Sém. Math. Sup., Vol. 102, Montreal, Presses Univ. Montréal, 1986, 141-184.

[7] Kupershmidt B.A., KP or mKP. Noncommutative mathematics of Lagrangian, Hamiltonian, and integrable systems, Mathematical Surveys and Monographs, Vol. 78, Providence, RI, American Mathematical Society, 2000 .

[8] Kupershmidt B.A., The quasiclassical limit of the modified KP hierarchy, J. Phys. A: Math. Gen., 1990, V.23, 871-886.

[9] Pommerenke C., Univalent functions, Göttingen, Vandenhoeck \& Ruprecht, 1975 (with a chapter on quadratic differentials by Gerd Jensen, Studia Mathematica/Mathematische Lehrbücher, Band XXV).

[10] Sato M., Soliton equations as dynamical systems on an infinite dimensional Grassmann manifold, RIMS Kokyuroku, 1981, V.439, 30-46.

[11] Sato M., Noumi M., Soliton equations and the universal Grassmann manifolds, Sophia University Kokyuroku in Math., Vol. 18, Tokyo, Sophia University, 1984 (in Japanese).

[12] Sato M., Sato Y., Soliton equations as dynamical systems on infinite dimensional Grassmann manifold, in Nonlinear Partial Differential Equations in Applied Science, Proceedings of the U.S.-Japan Seminar (1982, Tokyo), Lect. Notes in Num. Anal., 1982, V.5, 259-271.

\footnotetext{
${ }^{3}$ In [2] the system (B.2) $(\alpha=0)$ is called the "discrete KP", but such a name is used more often for the system with discrete independent variables. So we call (B.2) the "difference operator formalism" of the cmKP hierarchy.
} 
[13] Takasaki K., Initial value problem for the Toda lattice hierarchy, Adv. Stud. Pure Math., Vol. 4, Group Representations and Systems of Differential Equations (1982, Tokyo), Amsterdam, North-Holland, 1984, 139-163.

[14] Takebe T., Toda lattice hierarchy and conservation laws, Comm. Math. Phys., 1990, V.129, 281-318.

[15] Takebe T., Representation theoretical meaning of the initial value problem for the Toda lattice hierarchy. I, Lett. Math. Phys., 1991, V.21, 77-84.

[16] Takebe T., Representation theoretical meaning of the initial value problem for the Toda lattice hierarchy. II, Publ. RIMS, 1991, V.27, 491-503.

[17] Takebe T., A note on modified KP hierarchy and its (yet another) dispersionless limit, Lett. Math. Phys., 2002, V.59, 157-172, nlin.SI/0111012.

[18] Teo L.-P., On dispersionless coupled modified KP hierarchy, nlin.SI/0304007.

[19] Teo L.-P., Analytic functions and integrable hierarchies - characterization of tau functions, Lett. Math. Phys., 2003, V.64, 75-92, hep-th/0305005.

[20] Takasaki K., Takebe T., SDiff(2) KP hierarchy, in Infinite Analysis, Part A, B (1991, Kyoto), Adv. Ser. Math. Phys., Vol. 16, River Edge, NJ, World Sci. Publishing, 1992, 889-922, hep-th/9112046.

[21] Takasaki K., Takebe T., Integrable hierarchies and dispersionless limit, Rev. Math. Phys., 1995, V.7, 743808, hep-th/9405096.

[22] Takasaki K., Takebe T., Löwner equations and dispersionless hierarchies, in the Proceedings of XXIII International Conference of Differential Geometric Methods in Theoretical Physics Nankai Institute of Mathematics (August 2005, Tianjin, China), to appear, nlin.SI/0512008.

[23] Ueno K., Takasaki K., Toda lattice hierarchy, Adv. Stud. Pure Math., Vol. 4, Group Representations and Systems of Differential Equations (1982, Tokyo), Amsterdam, North-Holland, 1984, 1-95. 